\title{
Weitere Untersuchungen über die von einzelnen Organen - hervorgebrachten Substanzen mit spezifischer Wirkung.
}

\author{
VI. Mitteilung.
}

Von

Emil Abderhalden und Ernst Gellhorn.

(Aus dem physiologischen Institut der Universität Halle a. s.)

Mit 22 Textabbildungen.

(Eingegangen am 15. September 1921.)

Dic folgende Mittcilung knüpft unmittelbar an dje früheren und insbesondere an clie fünfte ${ }^{7}$ ) an. Wie clort, so wurke anch hier der Versuch unternommon, weitere für bestimmte Inkretstoffe spezifisehe Wirkungen festzustellex, um einen gangbaren Weg zu Isolierungsversuchen der wirksamen Stoffe aufzufinden. Die Versuche sind mit Optonen, die bei den folgenden Untersurhungen auf zwei Arten gewonnen worden sind, ausgeführt worden. Eincrseits wurden Organe, die Inkretstuffe hervorbringen, derch Fermente ticf abgebaut, andererseits wurde zur Hyrdrolyse 10 proz. Schwefoldälure verwendet. Die Wirkung der anf diesem Wegen gewomnenen Produkte wurde untereinander verglichen. Als Versuchsobjekt dienten: das Herzstreifenpräparat nach Loewe, das isolierte Frowchherz nach straub, ckrr Oesophagus vom Frosch und endlich seine Schwimmihaut. Die er'haltenen Befunde sind bei den dinzclnen Versuclben angeführt.

Es kann natïrlich nicht befriedigen, an einzelnen Organen bestimmte Wirkungen zu studieren, die von Produliten hervorgebracht werden, die von bestimmten Organen oder Geweben herstammen. Das Endziel allex dieser. Studien ist einerseits Anhaltspunkte für besonders charakteristische Wirkungen aufzufinden, $n m$ so in die Lage zu kommen, biologische Reaktionen auf bestimmte Inkretstoffe als Fahndungsmittel auf solche benützen zu können. Ferner sollen an Hand der gefundenen Erscheinungen nethe Gesichtspunkte zu Studien über die Wirkung dor Inkreistoffe im gesamten Organismus gewonnen werden. In dieser Richtung bedeuten die Studien über die Beeinflussung der Adrenalinwirkung auf das Blutgefäßsystcm und insbesondere auf die Blutcapillaren bereits einen erfolgreichen Vorstoß. Im Mittelpunkt des Interesses an den Funktionen des gesamten Kreis-

1) Pflügcrs Arch. f. d. ges. Physiol. 18\%, 243. 1921. 
laufes steht zur Zeit die Frage nach der Selbständigkeit des Capillarkreislaufes. Immer mehr sind Beobachtungen bekannt geworden, die für eine solche sprechen. Es sei an die Untersuchungen von Dale ${ }^{\mathbf{1}}$ ), Richards und Laidlaw, Ebbecke ${ }^{2}$ ) und $\mathrm{Krogh}^{3}$ ) erinnert. Sie sprechen im Sinne einer chemischen Regulierung des Capillarkreislaufes, sei es nun, daß bestimmte Produkte direkt auf die Capillarwand als Reizmittel in positivem oder negativem Sinne wirken, sei es, daß es sich um die Auslösung von Reflexen handelt. Unsere Beobachtungen legen den Schluß sehr nahe, daß es Inkretstoffe sind, die regulierend a uf den Capillarkreislauf einwirken. Es wäre damit eine neae Funktion der Inkretstoffe bildenden Organe festgestellt. Offenbar handelt es sich um ein Zusammenwirken mehrerer Inkretstoffe, die in feinsten quantitativen Abstufungen allen Ansprüchen, die an den Capillarkreislauf unter verschiedenen Bedingungen gestellt werden, gerecht werden. Es erscheint uns von ganz besonderem Interesse, daß a us der Schilddrüse, der $\mathrm{Hy}$ yophyse, der Thymus und den Generationsorganen stammende Produkte die Adrenalinwirkung auf Capillaren aufuheben vermögen. Sicherlich beschränkt sich die Wirkung bestimmter Inkretstoffe nicht nur auf die Capillaren, vielmehr werden auch die Arterien beeinflußt. Besonders bedeutungsvoll ist die Beobachtung, daß bei örtlicher Anwendung von Optonen auf der Schwimmhaut des Frosches eine augenblickliche Erweiterung von Capillaren feststellbar war, ferner vermehrte sich ihre Zahl deutlich. Diese Feststellungen ließen sich auch dann machen, wenn an größeren Arterien keine Veränderung der Gefäßweite zu erkennen war. Abb. 21 und 22 (vgl.\$. 78 u. 79) geben eine solche Beobachtung wieder. Sie zeigen das Aussehen der Froschschwimmhaut und zwar Abb. 21 nach Einwirkung von Adrenalin, während Abb. 22 den Einfluß von Adrenalin + Placenta - N. opton zeigt. Im letzteren Fall erkennt man ohne weiteres die stark erweiterten Capillaren. Im ersteren kann man die engen. Capillaren kaum erkennen.

Wir beabsichtigen diese Beobachtungen weiter $z u$ verfolgen und vor allem als Versuchsobjekte die Froschzunge und Harnblase heranzuziehen. Es dürfte die Feststellung, daß Inkretstoffe regulierend auf den Capillarkreislauf einwirken, nicht ohne weittragende Bedeutung für die Deutung des Einflusses der Organe mit Inkretion auf bestimmte Gewebsfunktionen sein. Gewiß ist die Funktion der einzelnen Gewebe bei bestimmten Inanspruchnahmen ganz wesentlich von der Durchblutung

1) H. H. Dale and Richards, Journ. of physiol. 52, 100. 1918. Dale and Laidlaw, ebenda p. 355.

2) U. Ebbeske, Pflügers Arch. f. d. ges. Physiol. 169, 1. 1917.

3) A. Krogh, Journ. of physiol. 52, 391, 409, 457. 1919. 53, 399. 1920. 
und insbesondere auch von der Tätigkeit der Capillaren abhängig. Ihre Beeinflussung durch Inkretstoffe dürfte dabei von ausschlaggebender Bedeutung sein. Manche Ausfallserscheinungen beim Versagen der Lieferung bestimmter Inkretstoffe, sei es, daß das Organ, das sie zu liefern hat, verändert ist oder ganz fehlt, läßt sich wenigstens wahrscheinlich in gewissem Umfange mit dem Versagen des feineren Capillarmechanismus in Zusammenhang bringen. Es wird von größter Bedeutung sein, diesen Gedankengängen bei der Beobachtung von inkretorischen Störungen nachzugehen. Man darf bei derartigen Überlegungen nie außer acht lassen, daß die bei Studien über die Wirkung von Inkreten angewandten Methoden recht grober Natur sind. Wir wenden die Inkretstoffe ohne Zweifel in viel zu großen Konzentrationen an. Wir lassen außer acht, daß wahrscheinlich die Wirkung der Inkretstoffe stets die Resultante des in feinster Weise abgestuften Zusammenspieles einer ganzen Reihe von solchen Stoffen ist. Der Ausfall des einen oder anderen Stoffes oder auch nur die quantitative Verschiebung in ihren Mengenverhältnissen kann schon bedeutungsrolle Folgen haben. Sie können aber auch von anderen Organen aus durch ähnlich oder gleich wirkende Inkretstoffe kompensiert werden. Man darf sich ohne Zweifel die Tätigkeit der sinzelnen Organc hinsichtlich ihrer innensekretorischen Funktion nicht so vorstellen, daß nun jedes Gewebe ausschließlich ganz andere Inkretstoffe bereitet. Vielmehr scheint der Fall so zu liegen, daß alle Organe oder doch viele davon gleiche oder doch ähnliche Wirkungen hervorbringende Produkte bilden und sehr wahrscheinlich darüber hinaus noch ein für sie spezifisches Inkret abgeben. Endlich darf nicht außer acht gelassen werden, daß für den Mechanismus der Regulation der Blutcapillaren nicht nur chemische Agentien in Frage kommen, die an irgendeiner Stelle peripher angreifen, vielmehr kann das Nervensystem mit eingreifen und scinerseits in dem Wechselspiel der einzelnen Reize seinen Einfluß geltend machen ${ }^{1}$ ). Man darf bei der Frage nach dem Mechanismus der Regulierung des Capillarkreislaufes nicht einseitig entweder chemische, peripher wirkende Stoffe in Betracht ziehen oder nervöse Einflüsse - seien sie nun zentral bedingt oder aber reflektorischer Art. Vielmehr muß man beide Arten von Einflüssen, nebeneinander und getrennt, in Betracht ziehen. Selbstverständlich besteht durchaus die Möglichkeit, daß auch die ,rein“ nervösen Mechanismen in engsten Beziehungen zu Wirkungen von Inkretstoffen stehen.

1) Vgl. hierzu E. Steinach und R. H. Kahn, Pflügers Arch. f. d. ges. Physiol. 9\%, 105. 1903. Bezüglich der Wirkungen psychischer Vorgänge auf den Kreislauf vgl. Ernst Weber, Der Einfluß psychischer Vorgänge auf den Körper. Berlin 1910 (daselbst Literatur) und E. Gellhorn und H. Lewin, Arch. f. Anat. a. Physiol. Physiol. Abt. 1913 u. 1915.

Pflügers Archiv f. d. ges. Physiol. Bd. 193. 
I. Versuche am Herzstreifenpräparat nach Löwe und am isolierten Froschherzen nach Straub.

In einer früheren Mitteilung ${ }^{1}$ ), in der das Herzstreifenpräparat unter verschiedenen Bedingungen untersucht wurde, hatte sich unter den in dieser Hinsicht geprüften Salzen fast a usschließlich Bariu mchlorid als wirksam erwiesen, die Automatie des nicht schlagenden Streifens anzuregen, gleichgültig, ob es sich um einen ganglienzellreichen Kammerlängsstreifen oder um eine ganglienzellarme Herzspitze handelt. Dabei zeigte sich, daß Bariumchlorid die bisher bekannten, zur Anregung der Automatie von Herzstreifen geeigneten Reizmittel [Dehnung ${ }^{2}$, Adrenalin] an Wirksamkeit bei weitem übertrifft. Es fragte sich nun, ob die von uns untersuchten Optone ebenfalls in diesem Sinne wirksam sind. Wir beschränkten uns hierbei nicht nur auf die Untersuchung der durch Autolyse bzw. fermentativen Abbau dargestellten Organpräparate (sog. Optone), sondern verwandten auch Extrakte, die durch $\mathrm{H}$ ydrolyse der Organe mit 10 proz. Schwefelsäure hergestellt waren. Sie sind durch ein , $\mathrm{S}^{\star *}$ gekennzeichnet. Alle untersuchten Optone, die in den verschiedensten Konzentrationen angewendet wurden, erwiesen sich mit Ausnahmedes Testishydrolysats (Testis $S$ ) als unwirksam. Daß das Fehlen der die Automatie anregenden Wirkung seitens aller Optone mit Ausnahme von Testis $\mathbf{S}$ nicht etwa daran lag, daB unerregbare Präparate verwendet wurden, konnte in jedem Versuche dadurch mit Sicherheit erwiesen werden, daß stets nach Ersatz der optonhaltigen Flüssigkeit durch frische Ringerlösung die Anregung der Automatie mit Bariumchloridlösung gelang. Wir müssen deshalb die Wirkung von Testis S a den nicht schlagenden Herzstreifen als eine spezifische ansehen. Was die wirksame Dosis von Testis $S$ anlangt, so genügte in allen Versuchen $0,01 \mathrm{~g}$ auf $60 \mathrm{ccm}$ Ringerscher Flüssigkeit. Als Belege mögen die Abbildungen 1 und 2 dienen. In Abb. 1 wurde an einem Kammerlängsstreifen, der in gut durchlüfteter Ringerscher Flüssigkeit suspendiert war, aber weder spontan noch auf Dehnungsreiz automatische Kontraktionen zeigte, bei $+20,01 \mathrm{~g}$ Testis S gegeben mit dem Erfolg, daß nach einer kurzen Latenzzeit regelmäßige Kontraktionen einsetzen, die auch nach Erneuerung der Ringerschen Flüssigkeit längere Zeit anhalten. Die Abb. 2 rührt von einem anderen Kammerlängsstreifen her, der nach Suspension in Ringerscher Flüssigkeit spontan sich regelmäßig kontrahierte. Nachdem diese Kontraktionen eine Reihe von Stunden ge-

1) Abderhalden und Gellhorn, Pflügers Arch. f. d. ges. Physiol. 183, 303. 1920 .

2) S. Löwe, Zeitschr. f. d. ges. exp. Med. 6, 300. 1918. 
währt hatten und in dieser Zeit verschiedene Salze in ihrer Einwirkung auf den schlagenden Kammerlängsstreifen studiert worden waren, stand der Kammerstreifen völlig still. Auf Dehnungsreiz wurde nur eine einmalige Kontraktion erzielt. Bei +44 wird $0,01 \mathrm{~g}$ Testis $\mathrm{S}$ der Ringerschen Flüssigkeit zugesetzt. Bald darauf setzen automatische Kontraktionen in Form von Lucia nischer Perioden ein, die auch nach Wechsel der Nährflüssigkeit noch andauerten. Die Tatsache, daß in diesem Versuche die Kontraktionen als Lucianische Perioden auftreten, findet wohl darin eine hinreichende Erklärung, daß der Herzstreifen durch zahlreiche Versuche bereits erheblich geschädigt war ${ }^{1}$ ). Auch an zahlreichen anderen Herzstreifenpräparaten, die verschiedenen Teilen des Froschherzens entnommen waren (Herzspitze, Kammerring-

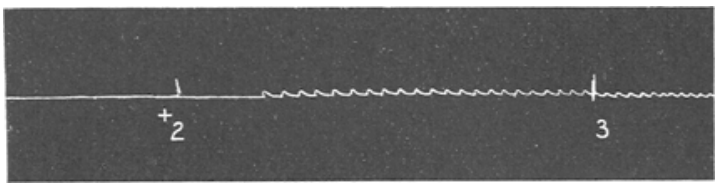

Abh. 1. Kammerlängsstreifen. Jei $+20,01 \mathrm{~g}$ Testinhydrolysat auf $60 \mathrm{ccm}$ Ringersche Flüsnigkeit. Bei 3 Wechsel der Ringerschen Flüssigkeit.

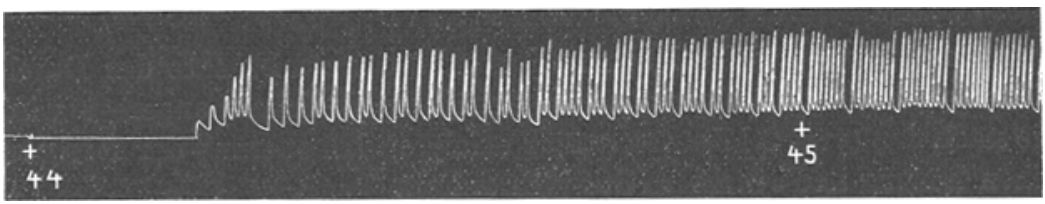

Abb. 2. Kammerlängsstreifen. Bei $+440,01$ g Testishydrolysat auf $60 \mathrm{ccm}$ Ringersche Flüasigkeit. Bei +15 Wechsel der Ringerschen Flüssigkeit.

streifen) gelingt es, den nicht schlagenden Herzstreifen durch Testis $A$ in Automatie zu versetzen. Allerdings muls betont werden, dal3 Bariumchloridlösung der Testis S-Optonlösung erheblich überlegen ist, da nicht selten an Präparaten, die auf Zusatz von Testis s nicht reagierten, Bariumchlorid dennoch die Automatie anzuregen vermochte.

Wir suchten nun die Wirksamkeit des Präparates zu steigern, indem wir zwecks Abtrennung unwirksamer Körper das Testishydrolysat einer fraktionierten Extraktion unterwarfen. Zunächst wurde auf dem Wasserbad am Rückflußkühler erschöpfend mit Alkohol extrahiert (12 Stunden), der Rückstand in wenig Wasser gelöst und abermals mit Alkohol extrahiert. Nunmehr wurden die Filtrate bei ciner Temperatur von $40^{\circ} \mathrm{im}$ Vakuum zur

1) Vgl. Abderhalden und Gellhorn, Pflïgers Arch. f. d. ges. Physiol. 183. 309 . 1920 . 
Trockene verdampft. Der Rückstand wurde in Wasser gelöst. Wir erhielten so ein alkoholisches Extrakt (I), ein alkoholisch wässeriges Extrakt (II) und, indem wir von dem letzteren abfiltrierten und den Rückstand in Wasser lösten, ein wässeriges Extrakt (III), Alle drei Extrakte wurden in 1 proz. Lösung $(1 \mathrm{ccm}$ auf $60 \mathrm{ccm}$ Ringersche Flüssigkeit) hinsichtlich ihrer Fähigkeit, die Automatie des nicht schlagenden Herzstreifens anzuregen, geprüft. Dabei zeigte sich, daß, während Extrakt I und II unwirksam waren, dem wässerigen Extrakt die erwartete Wirkung zukam und zwar in erheblich höherem Maße als dem wässerigen Hydrolysat, das noch nicht der Extraktion mit Alkohol unterzogen worden war. Daß selbst unter sehr ungünstigen Bedingungen diese Wirkung zustande kommt, lehrt Abb. 3, in der ein

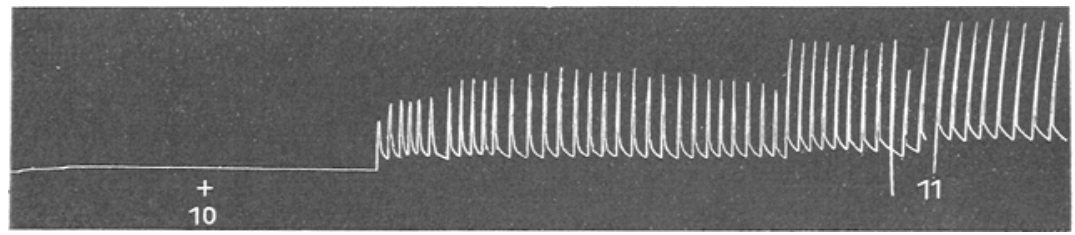

Abb. 3. Tiefer Kammerringstreifen ohne Durchlüftung. Bei $+100,01 \mathrm{~g}$ wäBriger Hodenextrakt III (cf. Text). Bei 11 Wechsel der Ringerschen Flissigkeit.

ganglienzellarmer, tiefer Kammerringstreifen trotz Fehlens der Durchlüftung der Nährflüssigkeit zu regelmäßiger Tätigkeit angeregt wird, die nach der etwa 3 Minuten später erfolgten Erneuerung der Ringer schen Lösung noch mehrere Stunden anhält.

Die Steigerung der Wirksamkeit des Extraktes III gegenüber dem nicht weiter behandelten Hydrolysat legt den Gedanken nahe, daß in dem alkoholischen Extrakt eine Substanz vorhanden war, die eine die Automatie des Herzstreifens hem mende Wirkung ausübt. In der Tat läßt sich zeigen (vgl. Abb. 4), daß das alko. holische Hodenhydrolysat $(0,01 \mathrm{~g}$ auf $60 \mathrm{ccm}$ Nährflüssigkeit) eine negativ inotrope und chronotrope Wirkung am schlagenden Herzstreifen entfaltet. Beide Wirkungen sind aber vollkommen reversibel, wenn man $(+13 \mathrm{Abb} .4)$ die gleiche Dosis.Extrakt III hinzufügt. Dieser Versuch wurde an zahlreichen Herzstreifen mit gleichem Erfolge wiederholt und gibt dadurch eine vollständige Erklärung für die größere Wirksamkeit des Extraktes III des Testishydrolysats gegenüber demselben Präparate vor Anwendung der Extraktion mit Alkohol.

Obwohl die Wirkung des Testishydrolysats wie des Extraktes III eine sehr charakteristische ist, so möchten wir doch betonen, daß die gleichsinnige Wirkung des Bariumchlorids weit stärker ist. Bei der Anwendung von Bariumchlorid sahen wir in weit über hundert Ver- 
suchen kaum je einen Versager, während bei besonders geschädigten Präparaten die Anregung der Automatie mit Testishydrolysat mißlang, nachträglicher Zusatz von Bariumchlorid aber regelmäßige Kontraktionen hervorrief.

In einer weiteren Versuchsreihe konnte der Nachweis erbracht werden, daß der die Automatie des Herzstreifens anregende Körper nicht etwa durch die Autolyse und den fermentativen Abbau, dem die Hoden zur Darstellung des Testisoptons $\mathrm{N}$ unterworfen waren, zerstört wird, sondern daß die Unwirksamkeit des Testisoptons (N) im Gegensatz zu dem durch Schwefelsäure erhaltenen Hydrolysat in dem nicht weit genug erfolgten Abbau begründet ist, oder aber, es wird eine hemmende Substanz beseitigt. Fs konnte nämlich in Versuchen an cler ganglienzellarmen Herzspitze gozeigt werden, daß das Testisopton $\mathrm{N}$. das nachträglich einer Hydrolyse mit 10 proz. Schwefelsäure unterworfen wird, in gleicher Weise wirksam ist wie das Testisopton S.

In Abb. 4 wird gezeigt, daß ein rlurch Extrakt I sowohl hinsichtlich seiner Chronotropie wie der Größe der Kontraktionen in negativem Sinne beeinfluBter Herzstreifen durch Testishydrolysat Extrakt III wieder die ursprüngliche Zahl und Größe der Kontraktionen erhält. Dieser Antagonismus ist aber kein spezifischer, vielmehr wird durch Zusatz von Extrakt III die Arbeitsleistung eines auch z. B. durch Sauerstoffmangel geschädigten Herzstreifens wesentlich erhöht. Eine derartige Beeinflussung ließ sich durch Testisopton, das durch Autolyse und Fermentwirkung abgebaut war, niemals erreichen. Vielmehr wurde mit diesem stets eine negative Einwirkung durch Verminderung der Pulsgröße und der Frequenz erzielt ${ }^{1}$ ).

Das Testishydrolysat reagiert in wässeriger Lösung neutral und ist ebenso wie Extrakt III völlig frei von Barium in ionisierter Form (keine Trübung bei Zusatz von Schwefelsäure). Um dem

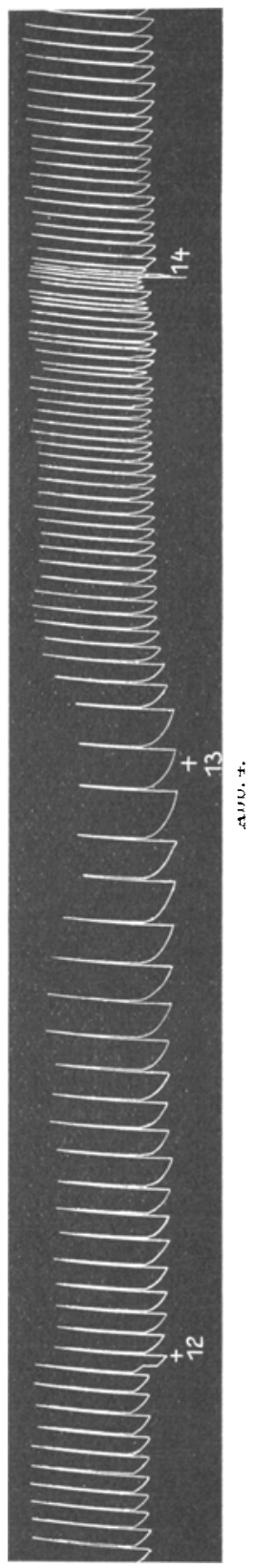

1) Vgl. hierzu Abderhalden und Gellhorn, Pflügers Arch. f. d. ges. Physiol. 18\%, $246 \mathrm{ff}$. 1921 . 
Einwand zu begegnen; daß das Barium komplex gebunden sein könnte und an seine Anwesenheit die charakteristische Wirkung des Testishydrolysats und des Extraktes III gebunden sei, wurde eine kleine Menge von Testishydrolysat und Extrakt III verascht, die Asche in Wasser gelöst und in verschiedenen Konzentrationen am Herzstreifenpräparat geprüt. Sie erwiesen sich alle als völlig wirkungslos. Damit dürfte der Beweis erbracht sein, daß Testishydrolysat und Extrakt III nicht anorganischen Beimengungen, sondern organischer Substanz ihre charakteristische Wirkung verdanken.

Durch weitere. Versuche suchten wir nun die Frage zu entscheiden, obdie a uf verschiedene Weise (durch Autolyse und Ferment-

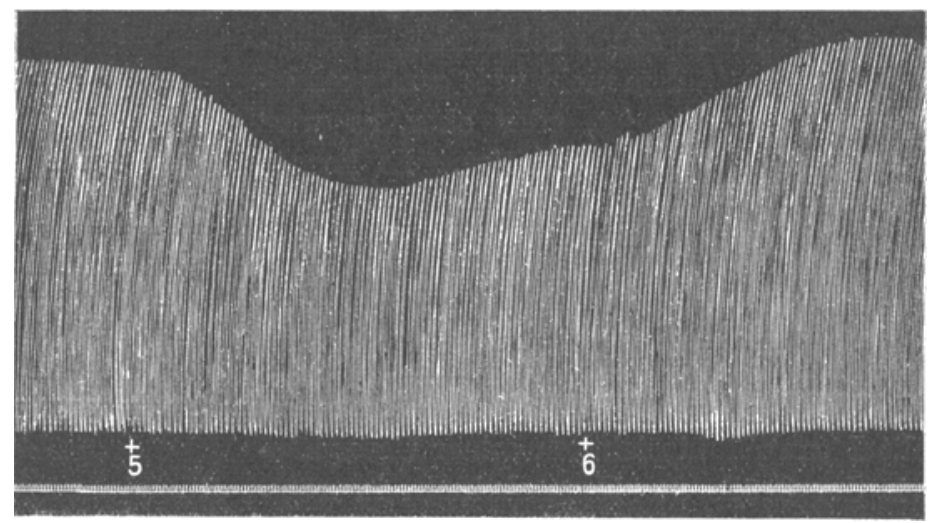

Abb. 5. Straubsches Präparat. Bei +5 Placenta N-Opton 0,002 g auf $1 \mathrm{ccm}$ Ringerscher Flüssigkeit. Bei +6 Wechsel der Ringersehen Flüssigkeit.

zusatz einerseits, durch Hydrolyse mit Schwefelsäure andererseits) erhaltenen optone gleichartige oder verschiedene Wirkungsbilder ergeben. Diese Untersuchungen wurden vergleichend an den Optonen von Testis, Schilddrüse und Placenta ausgeführt.

Zunächst seien die mit den Optonen aus Placenta angestellten Versuche geschildert. Wir müssen dabei auch auf die Wirkungen des durch fermentativen Abbau erhaltenen Placentaoptons ${ }^{1}$ ) eingehen, da die diesbezüglichen Versuche in unseren früheren Mitteilungen noch nicht enthalten sind.

Versuche am isolierten Froschherzen nach Straub ergeben (Abb. 5 und 6), daß Placenta $\mathrm{N}$ und $\mathrm{S}$ in 0,2 proz. oder stärkerer Lösung eine geringe Pulsverkleinerung bewirkt. Die Wirkungen sind

1) Statt Opton, durch Autolyse und Fermentzusatz dargestellt, sei der Kürze halber von Opton N im Gegensatz zu dem durch Hydrolyse mit Schwefelsäure erhaltenen Opton $S$ gesprochen. 
zbenso wie die der früher ${ }^{1}$ ) untersuchten Optone reversibel und zeigen iiemals Änderungen der Pulsfrequenz. Läßt man nun die Placentaoptone $\mathbf{N}$ und $\mathbf{S}$ längere Zeit einwirken, so zeigen sich ver-

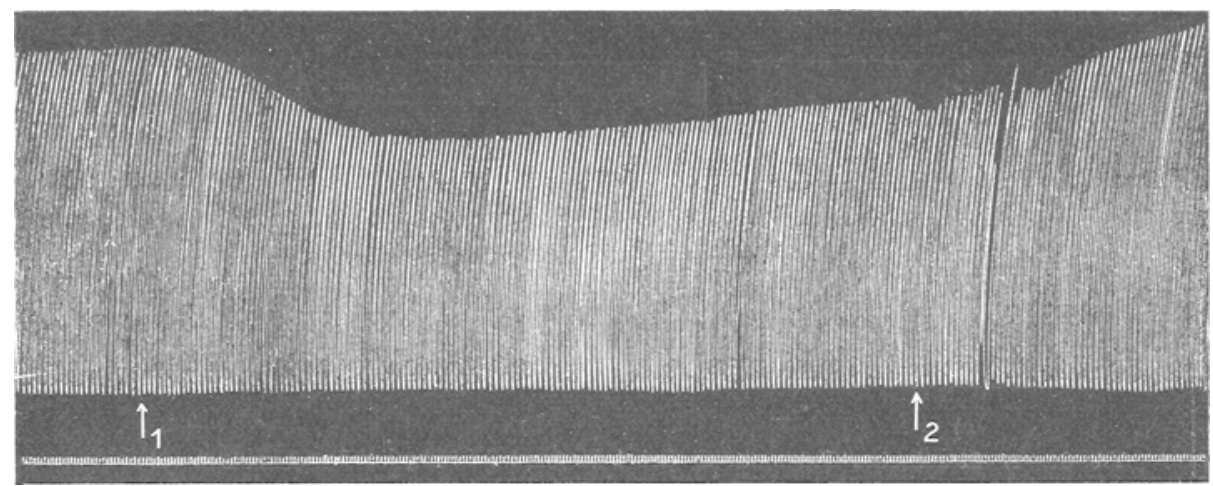

Abh. f. Straubsches ,Präparat. Bei 1 Placenta S-Opton 0,002 g auf 1 cem Ringersche Flüssigkeit. Bei 2 Wechsel der Ringersehen Flüssigkeit.

schiedene Ergebnisse. Bei Placenta $\mathbf{N}$ ist nämlich die Pulsver-

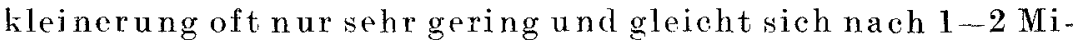
nuten von selbstaus: im Ansch] u 3 an diese Phase wird dann VergröBerung der Pulse beobachtet, die z. B. in dem Versuche der Abb. 7 fünf Minuten nach Zusatz von Placenta N (Abb. 7 b) ein

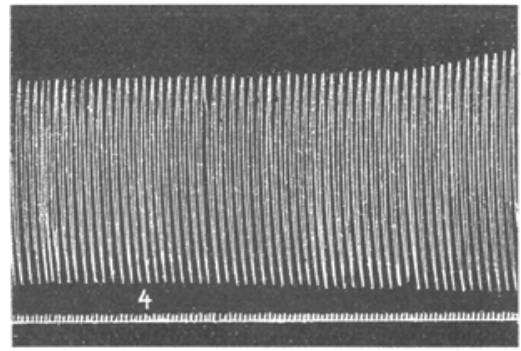

a

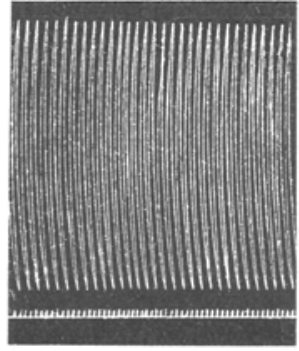

b

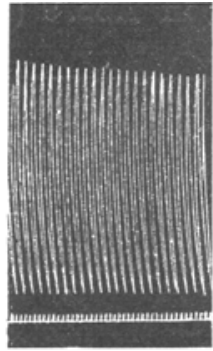

c)

Abb. 7 a-c. Straubsches Präparat. a) Bei $\neq$ Placenta $\mathbf{N} 0,002 \mathrm{~g}$ auf 1 com Ringer. b) Б3in. später. c) Nach Wechsel der Ringerschen Flüssigkeit.

Drittel der ursprünglichen Größe beträgt. Zuweilen wird die negativinotrope Wirkung gar nicht beobachtet, sondern es tritt nach einer Latenz von etwa einer Minute direkt die Pulsvergrößerung ein. Wechsel der Ringerschen Flüssigkeit führt wieder zu einer Abnahme der

1) Abderhalden und Gellhorn, Pflügers Arch. f. d. ges. Physiol. 182, 36 ff. 1920 und $18 \%, 248$. 1921. 
Pulsgröße, so daß diese sich der zu Beginn des Versuches bestehenden nähert. Placenta $S$ hingegen vermag niemals die Kontraktionen zu vergrößern, vielmehr hält die Abnahme der Puls. größe auch fünf Minuten nach Zusatz von Placenta S noch

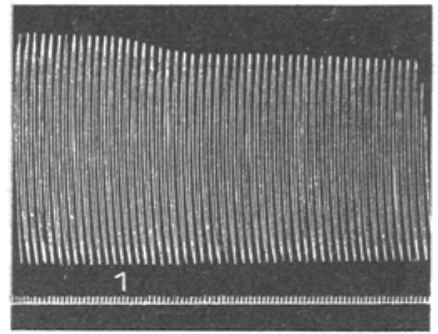

a)

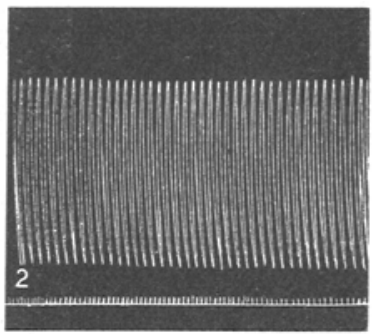

b)

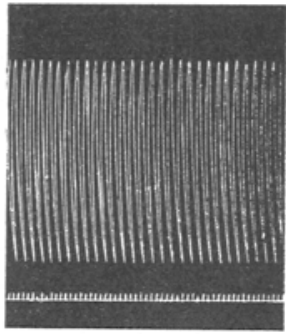

c)

Abb. 8a-c. Straubsches Präparat. a) Bei 1 Placenta $\mathrm{S} 0,002 \mathrm{~g}$ auf $1 \mathrm{ccm}$ 'Ringer. b) 5 Min. später. c) Nach Wechsel der Ringerschen Lösurg.

an (Abb. 8b) und erst die Erneuerung der Nährflüssigkeit vermag allmählich die ursprüngliche Kontraktionsgröße wiederherzustellen.

Es interessierte uns nun die Frage, ob in Analogie zu den Wirkungen, die die bisher untersuchten Optone am Herzstreifen ergaben, auch durch Placentaopton negativ chronotrope Wirkungen a uftreten können. Das ist nun in der Tat der Fall. In Abb. 9 (Kammerlängsstreifen) beobachtet man nach Zusatz von

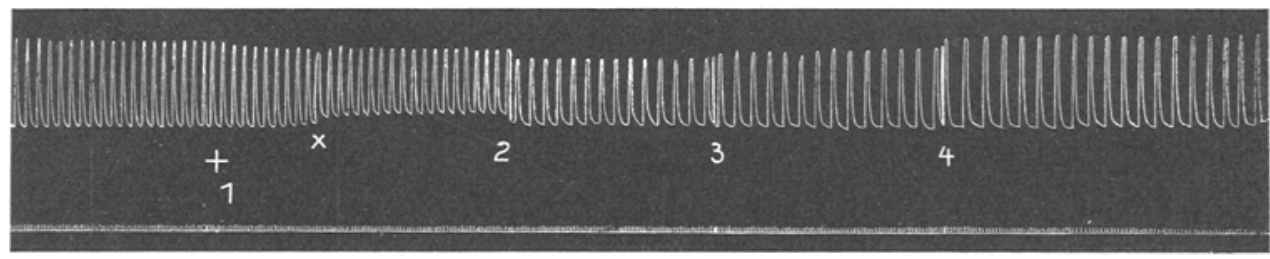

Abb. 9. Kammerlängsstreifen. Bei +1 Placenta $N 0,1 \mathrm{~g}$ auf $50 \mathrm{~cm}$ Ringersche Flüssigkeit. Bei $\times$ Kymographion für 1 Min. angehalten. Bei 25 Min. später. 3 und 4 je weitere 5 Min. später.

Placenta $\mathrm{N}(0,2 \%)$ eine geringe Pulsverkleinerung, allmählich kommt eine Pulsverlangsamung hinzu, die während des ganzen Versuches in etwa gleichem Maße beibehalten wird, während gleichzeitig die Pulsverkleinerung verschwindet und einer Vergrößerung der Pulse (Abb. 9, 4) weicht. Noch bedeutend stärker im Sinne der Verlangsamung ist am Herzstreifen die Wirkung von Placenta-S-Opton in Abb. 10. Hier führt sie $(0,2 \%)$ zu unregelmäßigen, seltenen Kontraktionen, schließlich zum Stillstand in Diastole. Es genügt jedoch, lediglich die Nähr- 


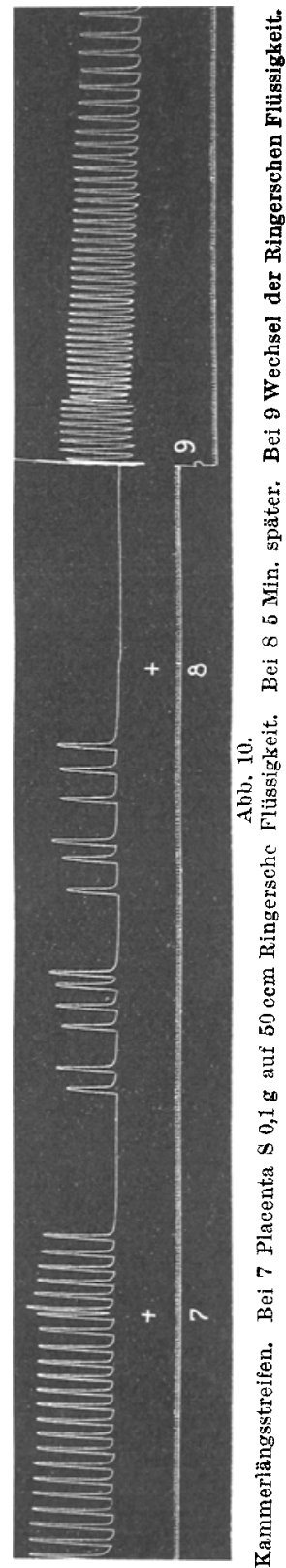

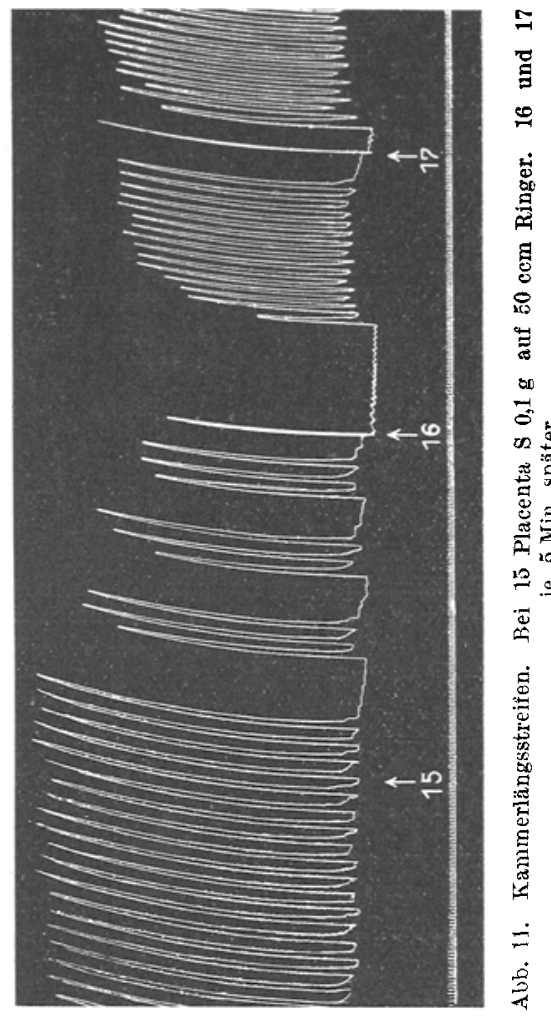

flüssigkeit zu erneuern, um wieder regelmäßige Kontraktionen von hoher Frequenz zu erhalten (Abb. 10;9). In einem anderen Versuche (Abb. 11) entstehen durch Zusatz von Placenta S Luciani sche Perioden von verminderter Pulsgröße. Dic Abb. 12 und 13 stellen je einen Versuch dar, in dem dic Optone der Placenta an dem Herzstreifen nach Amsler und $\mathrm{Pick}^{\mathbf{1}}$ ) geprüf werden. Dic obere Kurve rührt von der linken Herzkammerhälfte her, die noch mit Sinus und Vorhof in Verbindung steht, die untere verzeichnet die Kontraktionen der rechten Herzkammerhälfte. In beiden Kurven erkennen wir, daß Placenta $\mathrm{N}$ und $S$ an dem in Verbindung mit den übergeordneten Zentren stehenden Kam -

1) C. Amsler und E. P. Pick, Pflügers Arch. f. d. ges. Physiol. 184, 62. 1920. Vgl. auch Abderhalden u. Gellhorn, Pflügers Arch. f. d. ges. Physiol. 187, $249 \mathrm{ff} .1921$. 


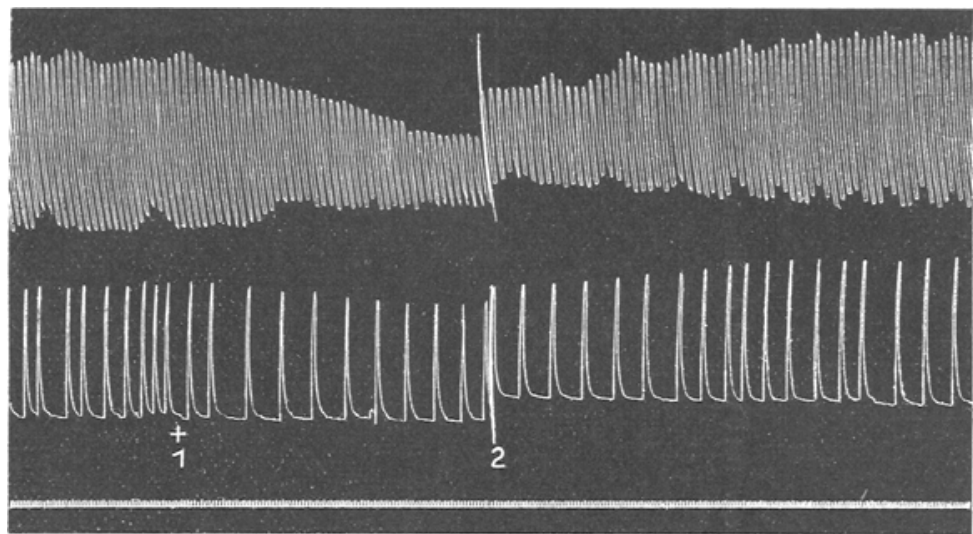

Abb. 12. Herzstreifenpräparat nach Amsler und Pick. Obere Kurve: Linke Herzkammerhälfte + Vorhof + Sinus. Untere Kurve: Rechte Herzkammerhälfte. Bei +1 Placenta S-Opton 0,1 g auf $50 \mathrm{ccm}$ Ringer. Bei 2 Wechsel der Ringerschen Flüssigkeit.

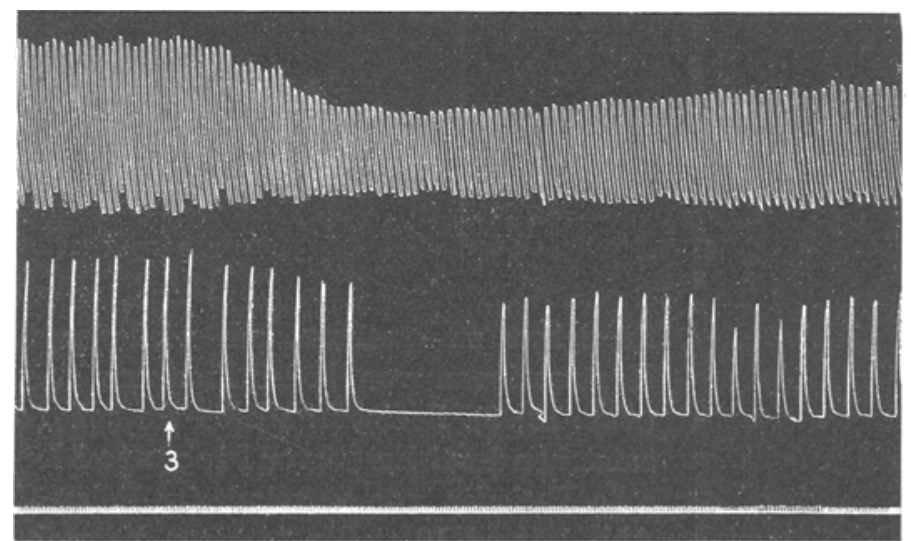

Abb. 13a. Herzstreifonpräparat nach Amsler und Pick (wie Abb. 12). Bei 3 Placenta N-Opton $0,1 \mathrm{~g}$ auf $50 \mathrm{ccm}$ Ringer.

merlängsstreifen lediglich die Pulsgröße verändern, während an dem isolierten Kammerstreifen (untere Kurve) die Pulsverlangsamung deutlich in Erscheinung tritt. Der Vergleich der Kurven 9 und 10 mit den Abb. 12 und 13 zeigt, daß bald Placenta $\mathrm{N}$ bald $\mathrm{S}$ die stärkste Pulsverlangsamung an dem isolierten Kammerstreifen hervorruft. Die Empfindlichkeit der Herzstreifen ist eben eine variable, gemeinsam aber ist allen Versuchen das qualitative Verhalten der Kammerstreifenkurve. Es geht also aus diesen Untersuchungen in völliger Übereinstimmung mit unseren 
früheren Ergebnissen ${ }^{1}$ ) hervor, daß Optone, die am isolierten Kammerstreifen die Pulsfrequenz verändern (negative Chronotropie), diese Wirkung am Gesamtherzen nach Straub ebenso wie am Kammerstreifen, der in Verbindung mit den primären Zentren steht, vermissen lassen ${ }^{2}$ ).

Die Untersuchungen am Herzstreifen haben also ergeben, daß $O p$ tone, die auf verschiedene Weise a us dem gleichen Organe hergestellt waren (Optone $\mathrm{N}$ durch fermentativen Abbau, Optone S durch Hydrolyse mit 10 proz. Schwefelsäure) verschiedene physiologische Wirkungen entfalten können. Die positiv inotrope Wirkung kommt nur dem Placenta $\mathrm{N}$ und nicht dem Placenta S-Opton zu. Außerdem ließ sich zeigen, daß Hydrolyse des Testis z u einem Präparat führt, das die Automatie des nicht schlagenden Herzstreifens anzuregen vermag, während das entsprechende N-Opton in dieser Hinsicht unwirksam ist. Es ergab sich nun die weitere Frage, ob auch an anderen Substraten den S und N-Optonen stets verschiedene

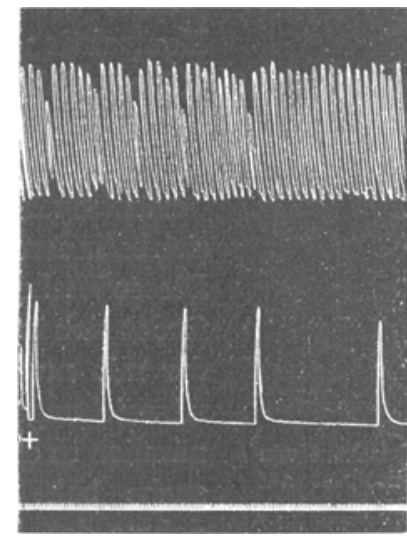

Abb. $13 \mathrm{~b}$.

3 Min. s]äter.

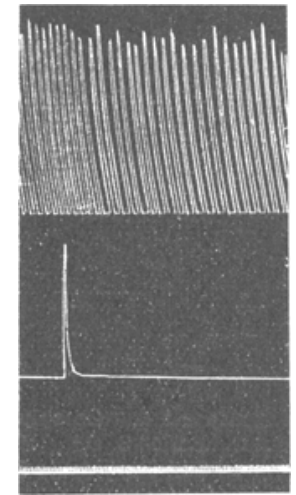

Ahb. 13 c. Nach Werhsel der Ringerschen Flüssigkeit.

Wirkung zukommt, und welches Opton dicjenige substanz enthält, der wir die spezifische inkretorische Substanz zubilligen müssen. In dieser Richtung wurden Versuche mit den S- und N-Optonen von Testis, Thyreoidea und Placenta am Oesophagus des Frosches wie am Kreislauf (Beobachtung desselben in der Schwimmhaut) angestellt.

1) Vgl. Abderhalden und Gellhorn, Pflügers Arch. f. d. ges. Phy̧siol. 18\%, 257. 1921.

2) Bezüglich der Deutung sei auf unsero frühere Mitteilung rerwiesen. 


\section{Versuche am Oesophagus des Frosches.}

Die Methodik war wiederum die gleiche, wie sie früher von uns beschrieben wurde ${ }^{1}$ ). Unsere früheren Untersuchungen hatten ergeben, daß die durch fermentativen Abbau dargestellten Optone der Schilddrüse eine geringe, vorübergehende Verkleinerung der Kontraktionen des überlebenden Oesophagus und eine geringe Abnahme des Tonus hervorrufen. Eine sehr viel stärkere und entgegengesetzt verlaufende Wirkung zeigt Schilddrüsenhydrolysat in der gleichen Konzentration $(0,1 \mathrm{~g}$ a uf 50,0 Ringer). Zunächst zeigt sich eine geringe Verkleinerung der Kontraktionen ohne Änderung des Tonus, sodann setzt aber eine

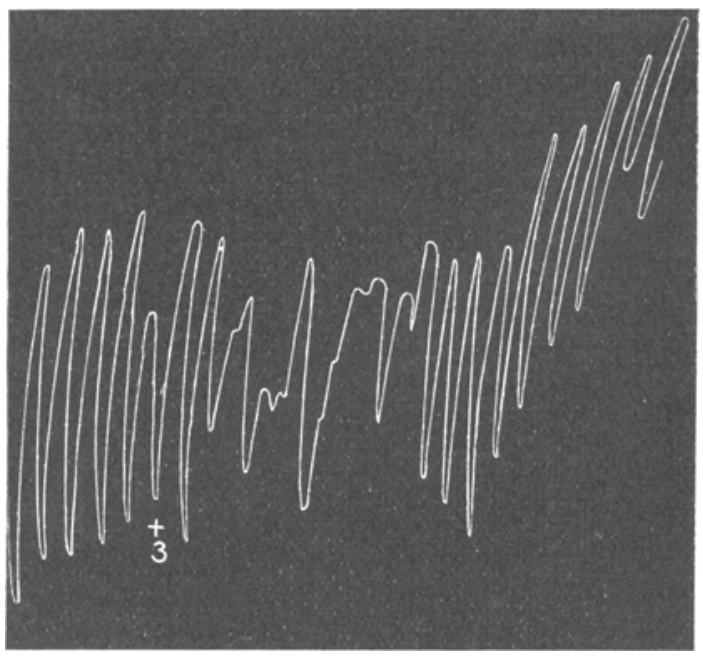

Abb. 14. Ringpräparat des Oesophagus mit Schleimhaut von Rana esculenta. Bei $+50,1 \mathrm{~g}$ Schilddrüsenhydrolysat auf $50 \mathrm{ccm}$ Ringersche Flüssigkeit. sehr bedeutende Zunahme des Tonus ein, die zur Folge hat, daß 2-3 Minuten nach $\mathrm{Zu}$ satz von Thyreoideahydrolysat die Fußpunkte der einzelnen Kontraktionen etwa in Höhe der Gipfelpunkte der Kurve vor Einwirkung des Schilddrüsenhydrolysates sich befinden (Abb.14). Dieser Wirkungstypus findet sich immer wieder mit nur geringen Abweichungen, indem nämlich in einem Versuche bald die Zunahme des Tonus in einem anderen Versuche bald die Vergrößerung der Kontraktionen überwiegt. Versuche mit Strumahydrolysat lehren, daß auch dieses erregend auf die Kontraktionen des überlebenden Oesophagus des Frosches einwirkt. Bevor aber die erregende Wirkung (Zunahme des Tonus, Vergrößerung der Kontraktionen) einsetzt, findet sich (Abb. 15) eine etwas längere negative Phase, in der der Tonus abnimmt und die Kontraktionen eine erhebliche Verkleinerung erfahren. Es hat die Kurve des Strumahydrolysats dadurch sehr viel Ähnlichkeit mit den stark erregend wirkenden Optonen (z. B. Corpus l uteum, Testis) erhalten, bei denen ebenfalls vor Eintritt der Tonus-

1) Pflügers Arch. f. d. ges. Physiol. 18\%, 257. 1921. 
zunahme ein mehr oder minder lang dauerndes Lähmungsstadium beobachtet werden konnte.

Die Optone der Placenta ( $N$ und $S$ ) zeigen in den Konzentrationen von 0,1 oder $0,05 \mathrm{~g}$ a uf 50,0 Ringersche Flüssig-

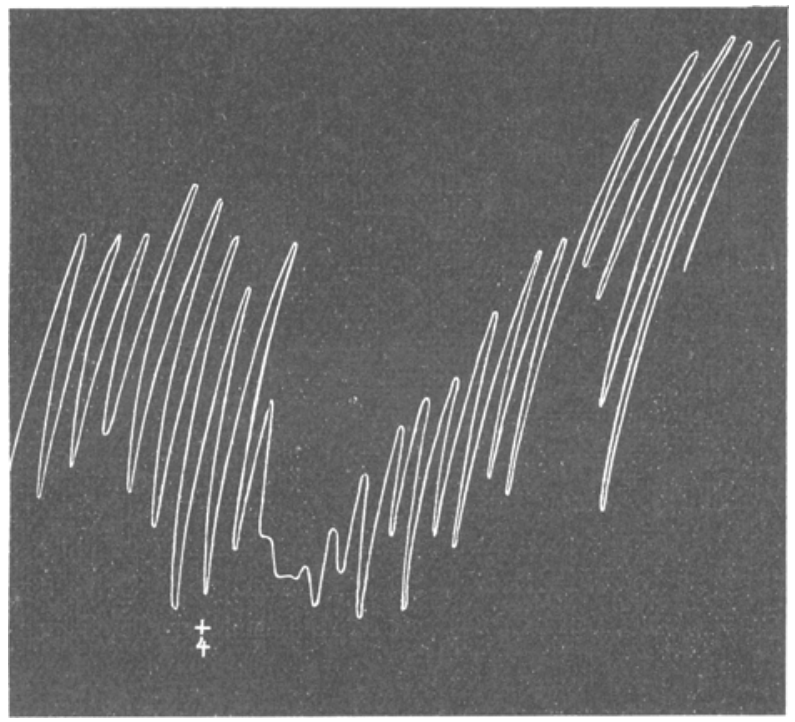

Abb. 15. Ringpräparat des Oesophagıs. Bei $+40,1 \mathrm{~g}$ Strumahydrolysat auf 50 ccm Ringersche Flüssigkeit.

keit, die die Scheidung der bisher untersuchten Optone in erregende und lähmende in Hinsicht auf die Beeinflussung der Automatie gestatteten, eine sehr starke lähmende Wirkung auf den überlebenden Oesophagus. Als Beispiel sei die Kurve der Abb. 16 wiedergegeben, in der der Zusatz von $0,1 \mathrm{~g}$ Placenta $\mathrm{N}$ die Automatie des Oesophagus völlig beseitigte. Placenta $\mathrm{S}$ zeigt genau die gleichen Wirkungen, so daß von der einer Wiedergabe der Kurven Abstand genommen werden kann. Aber auch diese Wirkungen sind völlig reversibel. Es genügt, die Ringersche

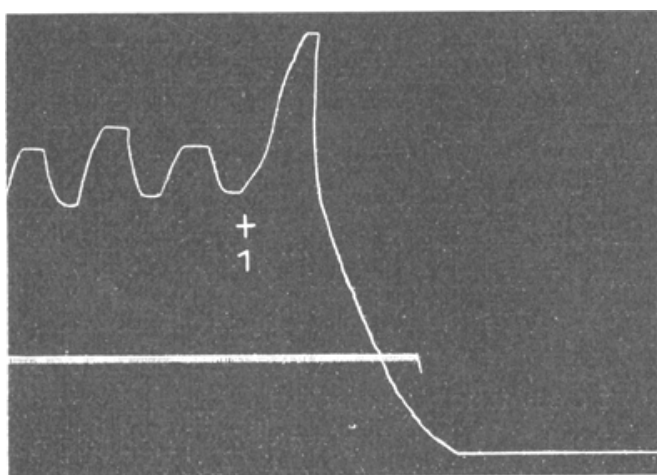

Abb. 16. Ringpräparat des Oesophagus. Bei +1 Placenta $\mathrm{N}$-Opton $0,1 \mathrm{~g}$ anf $50 \mathrm{ccm}$ Ringer. 
Flüssigkeit ein oder mehrere Male zu erneuern; um wieder normale Kontraktionen zu erhalten. Prüft man nun die Optone in der fünffach schwächeren Konzentration (0,02 auf 50,0 Ringer), so ergeben Placenta $\mathrm{N}$ und $\mathrm{S}$ verschiedene Resultate. Abb. 17 zeigt, daß Placenta $\mathrm{N}$

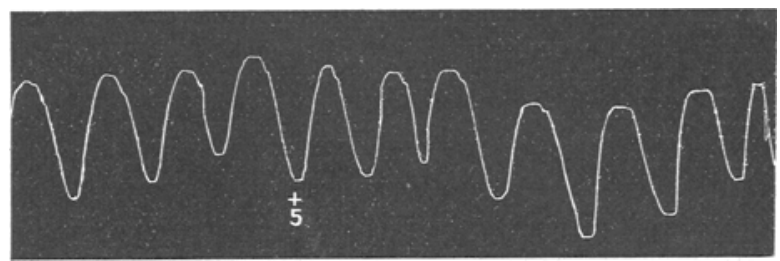

Abb. 17. Ringpräparat des Oesophagus. Bei +5 Placenta N-Opton $0,02 \mathrm{~g}$ auf $50 \mathrm{ccm}$ Ringer.

fast keine Veränderung der Kontraktionen hervorruft. Nur der Tonus nimmt vorübergehend in geringem Maße ab. Ist somit bei Placenta $\mathrm{N}$ noch eine geringe lähmende Wirkung angedeutet, so zeigt Placenta $S$ (Abb. 18) den entgegengesetzten Effekt. Die Kontraktionen nehmen nun etwa 100\% in Abb. 18 an Größe zu; die Wirkung ist aber nur eine vorübergehende, wie die letzten Kontraktionen der Abbildung bereits zeigen.

Die erregende Wirkung, die früher von Testisopton am Oesophagus des Frosches beschrieben wurde, kommt auch

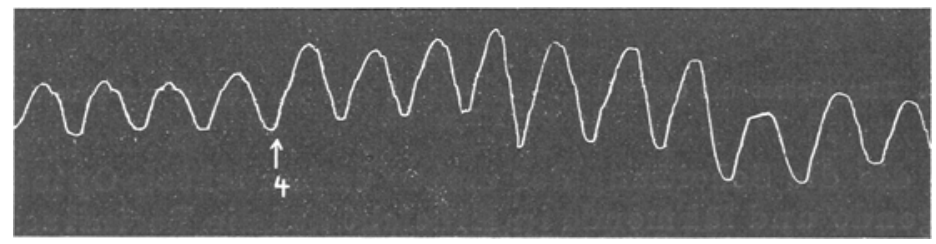

Abb. 18. Ringpräparat des Oesophagus. Bei +4 Placenta S-Opton $0,02 \mathrm{~g}$ auf $50 \mathrm{ccm}$ Ringer.

- in gleichen Konzentrationen - dem mit Schwefelsäure hydrolysierten Testisopton zu. Nur scheint es uns, als sei die Wirkung quantitativ erheblich stärker als die von Testis N. Für die außerordentlich starke Erhöhung des Tonus durch Testisopton $\mathbf{S}$ gibt Abb. 19 ein Beispiel.

Es war nun für uns von besonderem Interesse, festzustellen, ob dem Extrakt III, der aus hydrolysierten Testis nach Entfernung der alkohollöslichen Bestandteile hergestellt war (vgl. S. 52), eine von dem Testisopton $\mathbf{S}$ verschiedene Wirkung zukommt. Dies ist in der Tat der Fall. Es geht dies daraus hervor, daß es neben der erregenden Wirkung auf den überlebenden Oesophagusstreifen, diesich in nichts von der Wirkung von Testisopton $N$ oder $S$ unterscheidet, mit dem Extrakt III gelingt, die Automatie eines nicht schlagenden Streifens hervorzurufen, und zwar-unddaraus 

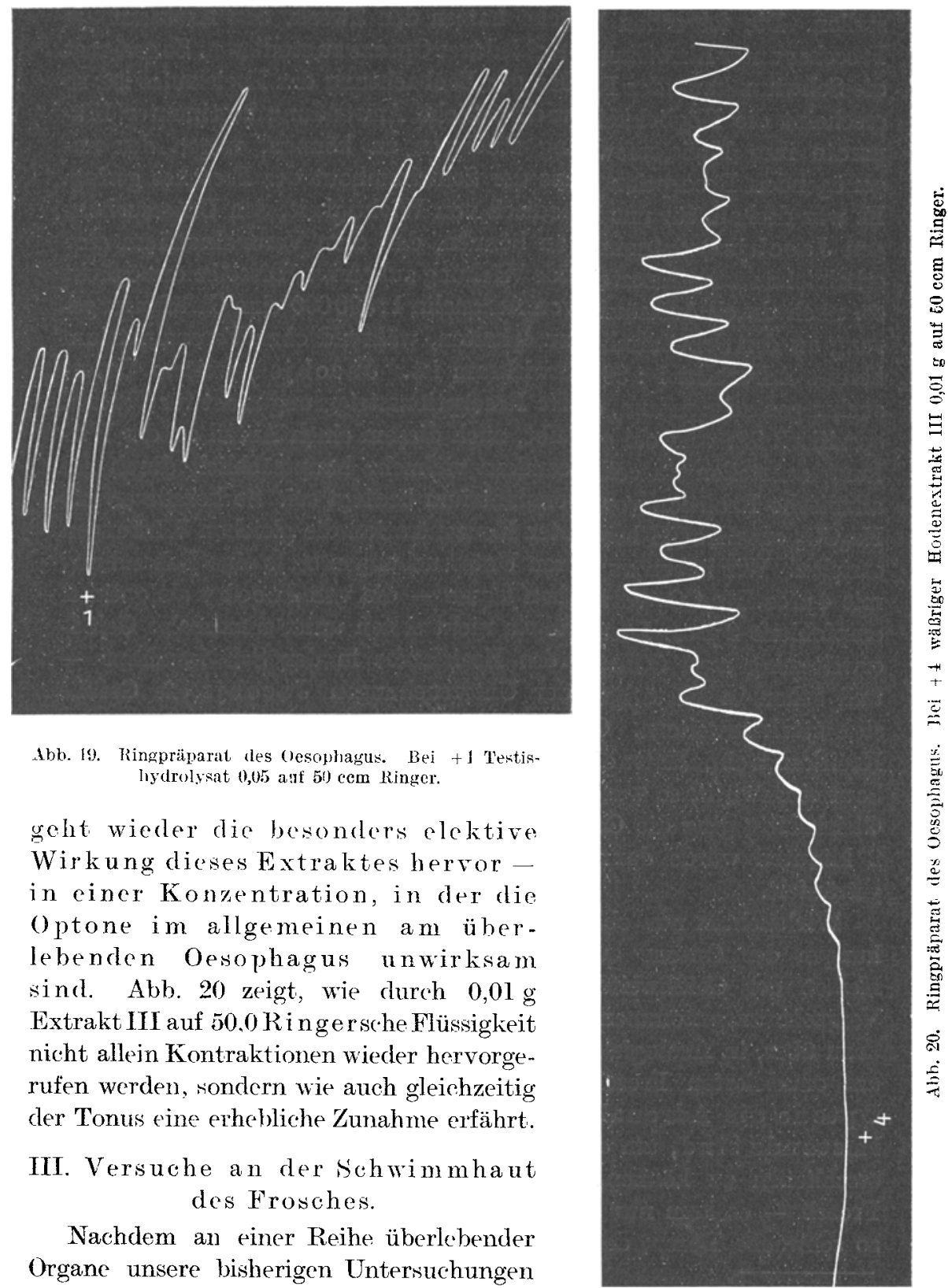

geht wieder die besonders elektive Wirkung dieses Extraktes hervorin einer Konzentration, in der die Optone im allgemeinen an über. lebenden Oesophagus nnwirksam sind. Abb. 20 zeigt, wie durch $0,01 \mathrm{~g}$ Extrakt III auf 50,0 Ringersche Flüssigkeit nicht allein Kontraktionen wieder hervorgerufen werden, sondern wie auch gleichzeitig der Tonus eine erhebliche Zunahme erfährt.

III. Versuche an der Schwimmhat des Frosches.

Nachdem an einer Reihe überlebender Organe unsere bisherigen Untersuchungen charakteristische Wirkungen der aus verschiedenen Organen mit innerer Seliretion dargestellten Optone ergeben hatten. waren wir bestrebt, ihre Bedeutung am Gesamtorganismus festzu- 
stellen. Die folgenden Versuche hatten den Zweck, den Kreislauf des Frosches unter Einwirkung verschiedener Optone zu studieren. So geeignet die Schwimmhaut des Frosches zur Beobachtung des Kreislaufes ist, so erscheint die Möglichkeit, durch örtliche Anwendung bestimmter Präparate ihre Kreislaufswirkung studieren zu können, wegen der ungenügenden Resorption seitens der Schwimmhaut des Frosches gering. Es war deshalb für uns methodisch von Wichtigkeit, den Untersuchungen $\mathrm{Jacobjs}^{1}$ ) entnehmen zu können, daß dem Veronalnatrium eine erhebliche resorptionssteigernde Wirkung zukommt. Dieselbe ermöglicht es, noch durch Aufträufeln von Adrenalin 1:500000 eine Gefäßverengerung an der Schwimmhaut, zu erhalten, während ohne Veronalnatrium bereits Adrenalin 1:3000 unwirksam ist. Jacobjs ${ }^{2}$ ) weitere Untersuchungen zeigten nun, daß die Resorptionssteigerung nicht für Veronal spezifisch ist, wiewohl er glaubt, auch diesem einen Anteil an der Wirkung zuerteilen zu sollen, sondern vielmehr hauptsächlich durch die alkalische Reaktion von Veronalnatrium bedingt sei. So konnte er mit $\mathrm{Na}_{2} \mathrm{CO}_{3}$ oder $\mathrm{NaOH}$ bestimmter Konzentration die gleichen Wirkungen erzielen. Wir haben zu unseren Untersuchungen stets eine Behandlung mit 4 proz. $\mathrm{Na}_{2} \mathrm{CO}_{3}$ vorausgehen lassen - die von $\mathrm{Jacob}$ y als optimal empfohlene 8 proz. Lösung führte nicht selten unter maximaler Gefäßerweiterung zum Stillstand des Kreislaufes in der Schwimmhaut und sahen in Bestätigung der Untersuchungen $J$ acobjs die Gefäße sich stark erweitern. Gleichzeitig trat auch eine Beschleunigung des Kreislaufes ein. Hatte die Sodalösung etwa 10 Minuten auf die Schwimmhaut eingewirkt, so wurde sie durch 0,6 proz. Kochsalzlösung abgespült. Gleichzeitig ging die Gefäßerweiterung allmählich zurück. Nach etwa 2 Minuten wurde dann die Optonlösung auf die Schwimmhaut aufgeträufelt.

Wir suchten nun ebenso wie Jacobj in seinen Versuchen die Wirkungen der Optone auf den Kreislauf in der Schwimmhaut durch Messen der Gefäßweite festzustellen. Da wir aber - ganz wenige Versuche ausgenommen - nur negative Resultate hatten, gingen wir dazu über, in Analogie zu den von uns früher beschriebenen Versuchen am enucleierten Froschbulbus ${ }^{3}$ ) die Frage zu entscheiden, ob die Optone die Adrenalinwirkung zu hemmen oder zu fördern imstande sind, um auf diese Weise indirekt ihre Kreislaufswirkung erschließen zu können. In diesen Versuchen curaresierten wir den Frosch - es kam nur Rana tem poraria zur Verwendung - durch so schwache Dosen, daß die Lähmung erst nach 30-45 Minuten ein-

\footnotetext{
1) Walther Jacobj, Arch. f. exp. Pathol u. Pharmakol. 86, 49. 1920.

2) Walther Jacabj, Arch. f. exp. Pathol. u. Pharmakol. 88, 333. 1920.

3) Abderhalden und Gellhorn, Pflügers Arch. f. d. ges. Physiol. 182, 28. 1920.
} 
trat. Auf diese Weise wird der Kreislauf, wie auch Klemensiewicz ${ }^{1}$ ) hervorhebt, fast gar nicht geschädigt. Sodann spannten wir die Schwimmhäute in der üblichen Weise aus und betrachteten sie durch zwei Mikroskope (Leitz Okular 4, Objektiv A). Zu den Versuchen kamen nur solche Frösche zur Verwendung, in deren beiden Schwimmhäuten der Kreislauf völlig gleichmäßig war. Außerdem suchten wir uns von der Richtigkeit der Optonwirkungen in jedem Falle, nachdem die Reversibilität ihrer Kreislaufswirkungen sich in zahlreichen Experimenten erwiesen hatte, nicht nur durch sehr häufige Wiederholungen der Versuche mit wechselnden Konzentrationen der Optone zu ïberzeugen, sondern wir sahen die Wirkung der Optone nur dann als bewiesen an, wenn es gelang, an demselben Frosch in zwei aufcinanderfolgenden Versuchen die Änderung der Adrenalinwirkung durch den Zusatz bestimmter Optone an beiden șchwimmbäuten in gleichem sinne anfzuweisen, während bald die eine bald die andere schwimmhaut zum Kontrollversuehe über die reine Adrenalinwirkung diente.

Tn den Tntersuchungen kamen die Optone a us Thyreoidea, Hypophyse, Ovar, Corpus luteum, Testis, Thymus and Placenta zur Verwendung und zwar bei Trestis und Placenta auch die durch Hydrolyse mit 10 proz. Schwefelsäure dargentellten Präparate.

Fs zeigte sirh, dals mit Ausnahme von Testis (N) und Placentat (S) sämtiche Optone, wenn auch in verschiedenem Grade, die Adrenalinwirkung zu hemmen vermögen.

Dies sei an der Hand e-iniger, verküryt wiedergegebener Protokolle. erläutert. Die linke bzw. rechte Spalte cles Protokolle "nthält die Be'nerkungen über die Kreislaufsveränderungen an der gleiehnamigen Schwimmhaut. Die dureh 4 proz. $\mathrm{Na}_{2} \mathrm{CO}_{3}$ bewirkte GefäBerweiterung und Kroislaufsbeschleunigung ist, weil sie regelmäßig eintritt, nicht weiter beschrieben worden.

Die Wirkung des Schilddrüsenoptons zeigt sich in folgendem Versuche:

Pan. tempor. Protokoll I.
$\left.10)^{\mathrm{h}}+5^{\prime} \quad 4^{0}, 0 \mathrm{Na}\right)_{2}\left(3_{3}\right.$.
$10^{\mathrm{h}} 35^{\prime} \quad 0,6^{\circ} ; \mathrm{NaCl}$.
10h $37^{\prime}$ Adrenalin 1:50000-f- Thyreo- ideapton $1 \%$.
$10^{11} 38^{\prime} \quad$ Beiderseits zeigt sich einc mäßige Verlangsamung der Zirkulation in Verbindung mit ciner Ver- engerung der arteriellen Gefäße.

$10^{\mathrm{h}} 39^{\prime} \quad$ Kreislauf wieder gebessert, fast normal,

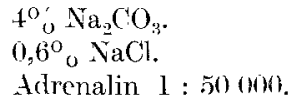

Völlige Puhe im ganzen Gesichtsfeld.

1) Klemensiewicz, Verfahren und Einrichtungen zur Beobachtung des Bhutstromes an Kaltblütern. Aus Abderhalden. Biologische Arbeitsmethoden.

Pflügers Arrhiv f. d. ges. Physiol. Bd. 1y;. 


\section{Ran. tempor. Protokoll I (Fortsetzung).}

$10^{\mathrm{h}} 41^{\prime} \quad$ In einem kleinen Teil des Gesichtsfeldes Stase; sonst fast normaler Kreislauf.

$10^{\mathrm{h}} 44^{\prime} \quad$ Keine Stase mehr vorhanden. Nur geringe Verlangsamung des Kreislaufes und minimale Gefäßverengerung.

$10^{\mathrm{h}} 48^{\prime} \quad$ Dasselbe.

$11^{\text {h }} \quad$ Fast normaler Kreislauf.

$11^{\mathrm{h}} 5^{\prime} \quad$ Kreislauf völlig wiederhergestellt. $11^{\text {h }} 8^{\prime} \quad$ Dasselbe.

$11^{\text {h }} 11^{\prime}$ Dasselbe.

$11^{\mathrm{h}} 2 \mathrm{O}^{\prime} \quad$ Dasselhe.

$11^{\mathrm{h}} 27^{\prime}$ Dasselbe.

$11^{\mathrm{h}} 30^{\prime} \quad 0,6^{\circ} \% \mathrm{NaCl}$.

$11^{\text {h }} 33^{\prime} \quad$ Normaler Kreislauf.

$11^{\text {h }} 35^{\prime}$ Dasselbe.

$11^{\text {h }} 39^{\prime}$ Dasselbs.

$11^{\text {h }} 41^{\prime} \quad$ Dasselb?.
Völlige Ruhe im ganzen Gesichtsfeld.

Dasselbe. Gefäße sehr stark verengt.

Völlige Ruhe.

Völlige Ruhe.

Völlige Ruhe.

In der sehr stark verengten großen Vene sehr langsame Zirkulation.

Sonst Ruhe.

In der Vene Zirkulation etwas schneller als $11^{\text {h }} 8^{\prime}$, auch in zwei Capillaren langsame, ruckweise Bluthewegung. Sonst Ruhe.

Im größten Teil des Gesichtsfeldes noch Ruhe. In der Vene und ihren venösen Zuflüssen Kreislauf gebessert, aber noch verlangsamt.

Auch in mehreren anderen kleinen Gefäßen langsame Zirkulation. Sonst wie $11^{\text {h }} 20^{\prime}$.

0,$6 ; \% \mathrm{NaCl}$.

Dasselbe wie $11^{\text {h }} 30^{\prime}$.

Kreislauf wesentlich gebessert. Gefäße weiter. Kreislauf noch verlangsamt, aber in allen Gefäßen vorhanden.

Weitere Besserung des Kreislaufs. Nur wenig langsamer als normal. Normaler Kreislauf.

Aus diesem Versuche geht hervor, daß im Kontrollversuche (rechte Schwimmhaut) nach Vorbehandlung mit 4 proz. $\mathrm{Na}_{2} \mathrm{CO}_{3}$ Adrenalin (H öchst) in einer Konzentration 1:50000 bereits nach 2 Minuten vollständigen Kreislaufsstillstand bewirkt, der erst eine halbe Stunde später $\left(11^{\text {h }} 8^{\prime}\right)$ allmählich zurückgeht. In der linken Schwimmhaut dagegen hat der Zusatz von 1 proz. Th yreoidea o pton bei gleicher Konzentration ${ }^{1}$ ) des Adrenalins nur eine unbedeutende, rasch vorübergehende Verlangsamung des Kreislaufes zur Folge. So kommt es, daß, während der Adrenalinkreislauf vollständige Stase im ganzen Gesichtsfeld aufweist,

1) Die Lösungen waren wie folgt zusammengesetzt:
a) Adrenalin $1: 25000$
b) Adrenalin 1:25000
Aq. dsst. āā.
Thyreoideaopton $2 \%$ ii . 
der mit Thyreoideaopton + Adrenalin behandelte Kreislauf der anderen Schwimmhaut ein annähernd normales Bild ergibt. Die Fortspülung der Adrenalinlösung und des Optons mit 0,6 proz. NaCl-Lösung führt nun auch an der rechten Schwimmhaut zur vollständigen Wiederherstellung des Kreislaufes; der bereits vorher völlig normale linke Kreislauf wird naturgemäß hierdurch nicht weiter beeinflußt.

Ran tempor. Protokoll II.

\section{$11^{\mathrm{h}}$}

$11^{\mathrm{h}} 10^{\prime}$

$11^{\text {h }} 11^{\prime}$

$11^{\mathrm{ln}} 15^{\prime}$

$11^{1+15^{\prime}}$

$11^{\mathrm{h}} 16^{\prime}$

$11^{\mathrm{h}} 17^{\prime}$

Il' Is' Dasselbe:

If $19^{\prime}$ Trotz Cefäßverengrimg noch im ganzen Gesichtsfedrl gutes Kreislauf.

$11^{1+} 21^{\prime} \quad$ Gefäbrorengerumg abgeklungen.
Kreislanf nomal.

$11^{11} 23^{\prime} \quad$ Kreislauf normal.

$11^{11} 25^{\prime}$ I assselbe.
Natr. carbon. $4 \%$.

$0,6 \% \mathrm{NaCl}$.

Adrenalin 1:50 (k).

Strömung stark verlangsamt, in der Arteric nur ruckweise Kirkulation.

Nur in den großen Venen langsame Zirkulation; in der Arterie und der (apillare völlige Ruhe.

Dic überwiogende Wehrzahl der (apillaren num kleineren Arteriolen jos nicht wahrnchmbar. Tr der giolsen Arterie mol cinem kleinen l'eil der rapillaren Wirderherinn der Zirkulation. Diese ist stark verlangsant und exfolgt rurkueise.

Weitere Besserung dos Kreislauts. In einem Teil der Capillaren besteht noch Ruhe.

Kreislauf fast normal, nur wenig verlangwamt.

Kreislanf normal.

In riesem Versuch wird dargetan, dak a uch die Vorbehandlung mil Schilddrüsenopton genügt, um rie Adronalinwirkung wesentich abzuschwächen. Zwar tritt auch in dem mit Thyreoirleaopton behandelten Kreislauf eine Gefäßrerengerung auf, cs liommt aber nirgends zu ciner stase und die Beweitigung der GefäBrerengerung und Kroislanfsverlangsamung erfolgt schneller als in dem Kontrollversuche.

\section{Ran. tempor. Protokoll III.}

$235^{\prime} \quad 4^{\prime \prime} ; \mathrm{Na}_{2} \mathrm{CO}$

$2^{14} 45^{\prime} \quad 0,6^{\prime \prime}, 0 \mathrm{NaCl}$.

2h $47^{\prime}$ Adrenalin 1: 50000 .
$40 \mathrm{Na,}(\mathrm{O})$

$0,6^{\circ}, \mathrm{NaCl}$.

Adrenalin 1 : 50000 - Thyreoideappton $0,1 \%$. 
Ran. tempor. Protokoll III (Fortsetzung).

$2^{\mathrm{h}} 48^{\prime} \quad$ Nur in den großen Venen langsame Zirkulation, sonst völlige Ruhe.

$2^{\mathrm{h}} 50^{\prime}, 3^{\mathrm{h}}$ und

$3^{\text {h }} 14^{\prime}$ Beiderseits völlige Ruhe.

$3^{\text {h }} 15^{\prime} \quad 0,6^{\circ} \% \mathrm{NaCl}$

$3^{\text {h }} 18^{\prime} \quad$ Völlige Ruhe im ganzen Gesichtsfelde.

$3^{\text {h }} 24^{\prime}$ u. $3^{\text {h }} 27^{\prime}$ Dasselbe.

$3^{\mathrm{h}} 28^{\prime} \quad 4^{\circ} \mathrm{Na}_{\mathrm{O}}^{\prime} \mathrm{Na}_{2} \mathrm{CO}_{3}$.

$3^{\text {h }} 29^{\prime} \quad$ Völlige Ruhe.

$3^{\text {n }} 30^{\prime}$ Langsamer Wiederbeginn der Zirkulation. In einem Teil der Gefäße noch Stase.

$3^{\text {h }} 30^{\prime} \quad$ Weitere Besserung der Zirkulation, Stase noch vorhanden.

$3^{\text {h }} 40^{\prime} \quad$ Zirkulation bedeutend schneller als $3^{\text {h }} 33^{\prime}$, aber noch langsamer als rechts. Nur zum geringen Teil noch Stase.

$3^{\text {h }} 50^{\prime} \quad$ Kreislauf fast normal.

$0,6 \% \mathrm{NaCl}$.

Kreislauf normal.

Dasselbe. $4 \% \mathrm{Na}_{2} \mathrm{CO}_{3}$.

Kreislauf stark beschleunigt, Gefäße etwas erweitert.

Dasselbe.

Dasselbe.

$4^{\mathrm{h}} 10^{\prime}$ Kreislauf normal.

Dasselbe.

Dasselbe.

Protokoll III zeigt endlich, daß sogar 0,1 proz. Thyreoideaopton noch eine dem Adrenalin entgegengesetzte Wirkung zu entfalten vermag. Hier führt zwar Adrenalin 1:50000 in beiden Schwimmhäuten zu vollständigem Kreislaufsstillstand. Aber in der rechten mit 0,1 proz. Thyreoideaopton vorbehandelten Schwimmhaut genügt die Fortspülung des Adrenalins durch Kochsalzlösung, um nach wenigen Minuten den Kreislauf völlig wiederherzustellen. Dagegen beharrte der nur mit Adrenalin 1:50000 behandelte Kreislauf auch 15 Minuten nach Abspülen der Adrenalinlösung durch physiologische Kochsalzlösung in vollständiger Ruhe. Erst durch Anwendung von 4 proz. Sodalösung, die an dem zuvor mit Thyreoideaopton behandelten Kreislauf der (rechten) Schwimmhaut zu einer starken Beschleunigung des Kreislaufes mit Erweiterung der Gefäße führt, gelingt es sehr allmählich den Kreislauf wiederherzustellen.

Aus den drei aus einer größeren Anzahl gleichartiger Versuche gewählten Protokollen über Versuche mit Schilddrüsenopton dürfte unzweifelhaft hervorgehen, daß Schilddr üsenopton bezüglich des Gefäßapparates eine gegenüber dem Adrenalin antagonistische Wirkung hervorbringt. Soweit die an der Sichwimmhaut des Frosches angestellten Versuche ein Urteil über das Ausmaß dieser Wirkung erlauben, scheint uns das Thyreoideaopton alle anderen gleichsinnig wirksamen Optone zu übertreffen. Denn es gelingt, auch direkt 
mit Schilddrüsenopton eine Gefäßerweiterung zu erzielen. Allerdings ist auch für diesen Nachweis die Resorptionssteigerung von seiten der Schwimmhaut mittels 4 proz. $\mathrm{Na}_{2} \mathrm{CO}_{3}$ unbedingt erforderlich. In einzelnen Versuchen konnten wir, gemessen am Okularmikrometer, eine Verdoppelung der Weite größerer arterieller Gefäße durch 5-10 proz. Schilddrüsenopton beobachten.

Auch dem Ovarialopton kommt eine deutliche Hemmung der Adrenalinwirkung zu.

Ran. tempor. Protokoll IV.
$4^{\text {h }} 35^{\prime} \quad 4 \% \mathrm{Na}_{2} \mathrm{CO}_{3}$.
$4^{\text {h }} 45^{\prime} \quad 0,6 \% \mathrm{NaCl}$.
$4^{\text {h }} 47^{\prime}$ Adrenalin 1: 100000 .
$4^{\text {h }} 48^{\prime}$ Die Arterie ist sehr stark verengt. Der Kreislauf im ganzen Ge- sichtsfeld verlangsamt, in eini- gen Capillaren bereits Stase.

$4^{\text {h }} 49^{\prime} \quad$ Nur in der großen Vene noch gute Zirkulation. Die Arteric ist fast nicht mehr sichtbax. In einem Teil der Capillaren Ntase, im auderen Teil sehr langsame, zum Teil ruckweise Bewegung.

$4^{\mathrm{l}} .51^{\prime}$ In der Vene noch lanesame Zirkulation, sonst völlige Ruhe in ganzen Gesichtsfeld.

$4^{\text {h }} .56^{\prime}$ Wiederbeginn der Zirkulation in der Articric. In der Vene noch verlangsamte Strömung, in ren Capillaren völlige Ruhe.

$5^{\mathrm{h}} 5^{\prime} \quad$ Kreislauf wesentlich gebesscrt, nirgends Stase, aber nooh langsamer als zu Beginn des Versuches. opton $2 \frac{1}{2} \%$.

Kreislauf verlangsamt, Gofäßc wenig verengt.

5) $37^{\prime}$ Kreislauf fast röllig wiederhergestellt sowohl hinsichtlich der sohnelligkeit der Blutbeweyung wie der (tefäßweitic.

.) $4 s^{\prime}$ Kroislauf tast normal.
$4 \% \mathrm{Na}_{2} \mathrm{CO}_{3}$

$0,6 \% \mathrm{NaCl}$.

Adrenalin 1:100000 + Ovarialopton $2^{1 / 2} \%$.

Kreislauf unverändert, vielleicht sogar etwas beschleunigt.

Dasselbo.

J)ascel be.

1)asselbe.

T)assolbo.

Dasselbe. $4^{\circ}, \mathrm{Na}_{2} \mathrm{CO}_{3}$. $0,6^{\circ} \% \mathrm{NaCl}$.

Adrenalin 1: 100000 .

Kreislauf verlangsamt.

Gefäße sehu stark verengt.

Dassclbe.

Gefäße stark verengt. In eirem Teil der Capillaren Stase, im ibrigen stark verlangsamte Zirkulation.

In cinem großen 'eil des (x'ssichtsfeldes noch Puht. In den größeren Gefäßen langsame, z. 'T. rucleweise Benregung. 
Ran. tempor. Protokoll IV (Fortsetzung).

\begin{tabular}{|c|c|c|}
\hline $5^{\mathrm{h}} 50^{\prime}$ & $0,6^{\circ} \% \mathrm{NaCl}$. & $0,6 \% \mathrm{NaCl}$ \\
\hline $5^{\text {h }} 52^{\prime}$ & Kreislauf völlig normal. & $\begin{array}{l}\text { Gefäße noch stark verengt. Zir- } \\
\text { kulation verlangsamt, z.T. Stase. }\end{array}$ \\
\hline $6^{\mathrm{h}}$ & Dasselbe. & $\begin{array}{l}\text { Kreislauf gebessert, aber noch } \\
\text { wesentlich verlangsamt. }\end{array}$ \\
\hline $6^{\text {h }} 10^{\prime}$ & Dasselbe. & $\begin{array}{l}\text { Nirgends Stase. Keine Gefäß- } \\
\text { verengerung; dagegen noch etwas } \\
\text { verlangsamte Strömung. }\end{array}$ \\
\hline
\end{tabular}

In diesem Protokoll ist ein Doppelversuch wiedergegeben, den wir sehr häufig zur Sicherstellung der Optonwirkung ausführten. Im ersten Versuch $\left(4^{\mathrm{h}} 35^{\prime}\right.$ bis $\left.5^{\mathrm{h}} 10^{\prime}\right)$ erfolgte der Zusatz des Ovarialoptons auf die rechte Schwimmhaut, im zweiten Versuche dagegen, der sich nach Wiederherstellung des Kreislaufes in beiden Schwimmhäuten dem ersten unmittelbar anschließt, auf die linke. Es wird -- bei gleichsinnigem Ausfall beider Experimente - auf diese Weise absolut sicher der Einwand entkräftet, daß der Kreislauf auch vor Beginn des Versuches nicht ganz gleichmäßig sei und deshalb durch den Adrenalinversuch eine ungleichmäßige Wirkung an beiden Schwimmhäuten zustande komme, die nicht auf Rechnung der Optone zu setzen sei.

Während Adrenalin 1: $100000+21 / 2$ proz. Ovarialopton im ersten Versuche kcine Kreislaufsänderung hervorruft, - die Adrenalinwirkung wird also vollständig paralysiert, - können wir im reinen Adrenalinversuche (linke Schwimmhaut) sehr schön die typische Beeinflussung des Kreislaufes durch schwache Adrenalinlösungen verfolgen. Es geht aus dem Protokoll hervor, wie die Verlangsamung des Kreislaufes vorwiegend die mittleren und kleineren Arterien betrifft, so daß in den Venen noch Zirkulation vorhanden ist, wenn in allen übrigen Gefäßen vollkommener Stillstand des Kreislaufes eingetreten ist. Entsprechend der relativ geringen Konzentration des Adrenalin geht seine Wirkung allmählich von selbst zurück und erlaubt nach abermaliger Verwendung von 4 proz. $\mathrm{Na}_{2} \mathrm{CO}_{3}$ die Wirkung des Ovarialoptons an der anderen Schwimmhaut zu studieren. Auch hier sehen wir, daß Adrenalin zu Gefäßverengerung und Kreislaufsverlangsamung führt, und daß in cinem großen Teil des Gesichtsfeldes jede Blutbewegung sistiert, während der Zusatz von $2^{1} / 2$ proz. Ovarialo pton an der linken Schwimmhaut die Adrenalinwirkung auf eine geringe, sehnell vorübergehends. Kreislaufsterlangsamung herabmindert.

Protokoll V.

Versuche mit Ovarialopton. Ran. tempor.

linke Schwimmhaut:

$10^{\text {h }} 51^{\prime} \quad$ Natr. carbon. $4 \%$.

$11^{\mathrm{h}} 1^{\prime} \quad 0,6 \% \mathrm{NaCl}$.

$11^{\mathrm{h}} 2^{\prime}$ Adrenalin $1: 20000+$ Ovarialopton 1\%:
Rechte Schwimmbant.

Natr. carbon. $4 \%$.

$0,6 \% \mathrm{NaCl}$.

Adrenalin 1:20000. 


\section{Protokoll V (Fortsetzung).}

$11^{\mathrm{h}} 3^{\prime} \quad$ Fast völlige Stase des ganzen Kreislaufes.

$11^{\text {h }} 5^{\prime} \quad$ Ǩreislauf im ganzen Gesichtsfeld fast normal. Nur in vereinzelten Capillaren noch Stase.

$11^{\text {h }} 6^{\prime} 30^{\prime \prime}$ Kreislauf völlig wiederhergestellt. Gefäße von normaler Weite.

$11^{\text {h }} 15^{\prime} \quad$ Normaler Kreislauf. $11^{\text {h }} 5^{\prime}$ Fast völlige Stase des Kreislaufes.

$11^{\text {h }} 5^{\prime}$ Völlige Ruhe im ganzen Gesichtsfeld.

$11^{\mathrm{h}} 7^{\prime}$ Dasselbe. Gefäße deutlich verengt.

$11^{\text {h }} 13^{\prime}$ Wiederbeginn des Kreislaufes in den großen Ger̈̈̈ßen. Die kleineren Arteriolen und Capillaren zeigen noch völlige Stase.

$11^{\text {h }} 15^{\prime}$ Nirgends Stase. Die Zirkulation ist deutlich gebessert, aber im ganzen noch verlang. samt.

Dieser Versuch zcigt, wie stärkeren Arrenalinlösungen gegenüber (1 : 20000$)$ Ovarialopton den völligen Kreislaufsstillstand nicht zu verhindern vermag. Seine Wirkung kommt aher in der berleutend sebnelleren Wiederherstcllung des Kreislanfes als im reinen Artrenalinversuch zum Austruck.

$$
\text { Ran. tempor. Protokoll VI. }
$$

\begin{tabular}{|c|c|}
\hline $4^{h}+2^{\prime}$ & $\left.4^{\prime \prime} \mathrm{Na}_{2} \mathrm{Cl}\right)_{3}$ \\
\hline $\left.4^{h} 55^{5}\right)^{\prime}$ & $0,6^{\circ}, 0 \mathrm{NaC}$ \\
\hline $4^{h} 55^{\prime}$ & $\begin{array}{l}\text { Idrenalin } 1: 75000=0 \text { (Narial } \\
\text { opton } 10 \% \text {. }\end{array}$ \\
\hline $4^{11}: 2 f^{\prime}$ & $\begin{array}{l}\text { (iefäbe etwas erweitert, Kreislauf } \\
\text { sonst völig nnveründert. }\end{array}$ \\
\hline $4^{11} .7^{\prime}$ & Danselbe. \\
\hline $4^{11} 5 \mathrm{~S}^{\prime}$ & $\begin{array}{l}\text { Kreisłauf stark velangsant: in } \\
\text { einem großen Teil des Gesiehts- } \\
\text { feldes völlige Ruhe. Nur in der } \\
\text { großen Vene noeh gute Zir- } \\
\text { kulation. }\end{array}$ \\
\hline
\end{tabular}

$4^{\circ}, \mathrm{Na}_{2} \mathrm{CO}$.

$0,6^{\circ} \mathrm{NaC}$.

Adrenalin $1: 75000$.

$4^{\text {h }} .56^{\prime}$ In der groben Arterie, die ad maximum verengt ist, Stase. In übrigen Zirkulation noch vorhanden, aber stark rerlangsamt.

$4^{12} 57 \mathrm{Nur}$ in der großen Vene und einigen Nebenästen dieser Venen langsame, z. 'I. ruckweise ' Zir'kulation. Im ibrigen Gesichtsfeld völige Aufhebung des Kreislaufes unter starker: Verengerung der arteriellen Gefälie.

$4^{\text {h }} 58^{\prime}$ Dassclbe.

Bemerkenswert an diesem Versuch ist, dabsofort uach ortheher Anwendung der Adrenalin-Ovarialoptonlösung cine Gefäbcrweiterung beobachtet wird, während im Adrenalinkontrollversuche sich maximale Gefäberengerung und Ver. 
langsamung des Kreislaufes einstellt. Die relativ geringe Wirksamkeit des Ovarialoptons in 1 proz. Lösung zeigt sich in diesem Versuche darin, daß es die Adrenalinwirkung nur zeitlich hinausschiebt, ohne die Intensität der Wirkung zu vermindern. Denn in diesem Versuche trat in beiden Schwimmhäuten vollständiger Stillstand des Kreislaufes ein, der erst durch Abspülen der Adrenalinlösung beseitigt werden konnte.

Der Vergleich der Stärke der Adrenalinwirkung in den Versuchen $\mathrm{V}$ und VI zeigt, daß bei den einzelnen Fröschen trotz gleicher Vorbehandlung mit 4 proz. $\mathrm{Na}_{2} \mathrm{CO}_{3}$-Lösung starke Verschiedenheiten in der Wirksamkeit des Adrenalins in Erscheinung treten. Deshalb beruhte auch eine Hauptschwierigkeit bei diesen Versuchen darin, eine Adrenalinlösung in Anwendung zu bringen, die weder zu stark war; so daß antagonistische Wirkungen der Optone verdeckt werden konnten, noch zu schwach, so daß keine deutlichen Unterschiede in den Kreisläufen der beiden Schwimmhäute sichtbar wurden. Ob der Tinterschied in dem ungleichen Verhalten der Frösche in einer ungleichen Empfindlichkeit für Adrenalin oder in einer verschieden großen Steigerung der Resorption durch 4 proz. $\mathrm{Na}_{2} \mathrm{CO}_{3}$ beruht, möchten wir noch unentschieden lassen.

Eine direkte Gefäßerweiterung durch Ovarialopton an der Schwimmhat des Frosches wurde nur in ganz scltenen Fällen und in geringem Ausmaße beobachtet.

Ran. tempor. Protokoll VII.
$10^{\mathrm{h}} 3^{\prime} \quad 4 \% \mathrm{Na}_{2} \mathrm{CO}_{3}$.
$10^{\mathrm{h}} 13^{\prime} \quad 0,6 \% \mathrm{NaCl}$.
$10^{\mathrm{h}} 15^{\prime}$ Adrenalin 1:50000+3\% Hypo- physisopton.
$10^{\mathrm{h}} 16^{\prime} \quad$ Kreislauf normal, unveründert.

$10^{\mathrm{h}} 17^{\prime}$ u. Dasselbe.

$10^{\text {h }} 19^{\prime}$

$10^{\mathrm{h}} 20^{\prime} \quad$ Dasselbe.

$10^{\text {h }} 24^{\prime}$ Dasselbe, vielleicht ein wenig verlangsamt.
$4 \% \mathrm{Na}_{2} \mathrm{CO}_{3}$.

$0,6 \% \mathrm{NaCl}$.

Adrenalin 1 : $50000 \%$.

Arterie völlig verengt, nieht mehr sichtbar. In einem Teil der Capillare. Stase des Kreislanfes. $10^{\mathrm{h}} 17^{\prime}$ u. $10^{\mathrm{h}} 19^{\prime}$ Völlige Rube mit Ausnahme der langsamen Zirkulation des Blutes in dex großen Vene und einem kleinen Seitenast derselben.

In einem Teil der Capillaren Wiederbeginn der Zirkulation. Die Bewegung des Blutes ist aber noch sehrlangsam und ruckweise. Die große Arterie ist noch ad maximum kontrahiert und deshalb fast unsichtbar.

Wiederbeginn der Zirkulation in der großen Arterie, die noch deutlich verengt ist. Capillarkreislauf gebessert, aber noch sehr langsam. 
Ran. tempor. Protokoll VII (Fortsetzung).

$10^{\mathrm{h}} 25^{\prime} \quad 0,6 \% \mathrm{NaCl}$.

$10^{\mathrm{h}} 27^{\prime} \quad$ Der Kreislauf ist beiderseits völlig normal.

$10^{\mathrm{h}} 54^{\prime} \quad 4_{\%}^{\mathrm{o}} \mathrm{Na}_{2} \mathrm{CO}_{3}$.

$11^{\mathrm{h}} 4^{\prime} \quad 0,6 \% \mathrm{NaCl}$.

$11^{\mathrm{h}} 6^{\prime} \quad$ Adrenalin $1: 50000$.

$11^{\text {h }} 7^{\prime} \quad$ Völlige Ruhe im ganzen Gesichtsfeld. Gefäße blutleer, sehr stark verengt.

$11^{\text {lh }} 8^{\prime} \quad$ Dasselbe.

I $1^{\text {b }} 11^{\prime}$ Pis auf minimale Bewegung des Blutes in einer kleinen vene völlige Ruhe.

$11^{\text {h }} 16^{\prime}$ Dasselbe. In der kleinen Vente nur zeitweilig ruckweiso BCwegung. Sonst völlige Ruhe. Gefäße noch verengt und blutleer.

$11^{h} 22^{\prime} \quad 0,6^{\circ} \% \mathrm{NaCl}$

$11^{\mathrm{h}} 23^{\prime}$ Allmäblicher Wirdorberginn der. Kirkulation. $0,6^{\circ} ; \mathrm{NaCl}$.

$4 \% \mathrm{Na}_{2} \mathrm{CO}_{3}$.

$0,6^{\circ}: \mathrm{NaCl}$.

Adrenalin I : $50000+$ Hypophysisopton $3 \%$;.

Kreislauf in den Capillaren und kleineren Gefäßen ein wenig verlangsamt. Keine Gefäßverengerung.

In einem Teil der Capillaren, die strotzend mit Blutkörperchen gefüllt sind, Stase. Im übrigen, besonders in den größeren Gefäßen gute, nur wenig verlangsamte Zirkulation.

Bis auf die geringe Stase in cinem kleinen Teil des Gesichtofeldes normaler Kreislaut.

Dasselhe; (refäBe sehr wut gefiillt.

$\left(9,6^{\prime \prime}, 0 \mathrm{Na}+1\right.$.

It $22^{\prime}$ Krristauf normal.

I $^{\text {h }} 333^{\prime}$ Kreislanf normal.

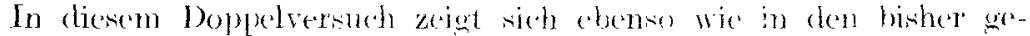
schilderten Versuchen. dak auch das Opton der Hypophese dite Adrenalinwirk ung $z u$ hemench i motande ist. Benerkenswert ist in dem zweiten Versuch. daßs deo Entersehied in der Weite der Gefäke in den beiden Schwimmbäuten stark hervortritt. Arrenalin allein erzeugt blutleere, maximal rerengte capillaren, während in dem Versuche mit Adrenalin + Hypophysisopton dis Capillaren weit und strolzend gefüllt sind. Tas gleiche Phänomen ist anch in dem Vor-

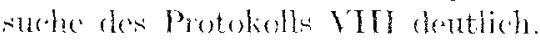

$$
\text { Lan. tempor. Prutokoll VIIl. }
$$

\begin{tabular}{|c|c|}
\hline $27^{\prime}$ & $4_{0}^{\circ} \mathrm{Na}_{3} \mathrm{CO}_{3}$. \\
\hline $3^{n} 15^{\prime}$ & $0,6^{\circ} \mathrm{NaCl}$ \\
\hline${ }^{1} 1$ & $\begin{array}{l}\text { Adrenalin } 1: 70000+\mathrm{H}_{y} \text { pophy } \\
\text { sisopton } 1 \% \text {. }\end{array}$ \\
\hline$]$ & $\begin{array}{l}\text { Beiderseits starke Verlangsamung } \\
\text { des Kreislaufes. Im größten Teil } \\
\text { des Gesichtsfeldes keine Blut- } \\
\text { bewegung. In den großBen Vencon } \\
\text { rhythmische Blutbewegung. }\end{array}$ \\
\hline
\end{tabular}

$4 \% \mathrm{Na}, \mathrm{CO}_{3}$

$0,6^{\circ} \mathrm{NaCl}$.

Artrenalin $1: 75000$.

derseits starke Verlangsamung des Kreislaufes. Im größten Teil bewegung. In den großen Venen ithythmisehe Blutbewegung. 
74 E. Abderhalden n. E. Gellhorn: Woitere Untersuchungen üher ،lic

Ran. tempor. Protokoll VIII (Fortsetzung).

$3^{\text {h }} 22^{\prime} \quad$ Beiderseits fast völliger Stillstand der Blutbewegung. Capillaren strotzend mit Blut gefüllt.

$3^{\mathrm{h}} 25^{\prime} \quad$ Zirkulation in der großen Vene gebessert. Gefäßerweiterung und Blutfüllung unverändert. Fast überall völlige Ruhe.

$3^{\text {h }} 29^{\prime}$ Wiederbeginn der Zirkulation in einem Teil der übrigen Gefäße.

$3^{\text {h }} 33^{\prime} \quad$ In der Hälfte der Capillaren noch Stase. In den übrigen Gefäßen weitere Besserung der Zirkulation.

$3^{\text {h }} 40^{\prime} \quad$ Nur vereinzelt noch Stase; sonst normale, nur wenig verlangsamte Zirkulation.

$3^{\text {h }} 44^{\prime} \quad$ Fast normaler Kreislauf, nirgends Stase.

$3^{\text {h }} 50^{\prime} \quad$ Dasselbe. $0,6 \% \mathrm{NaCl}$.

$3^{\text {h }} \mathbf{5 4 ^ { \prime }} \quad$ Normaler Kreislauf.

Capillaren und auch größere arterielle Gefäße blutleer und so stark kontrahiert, daß sie kaum sichtbar sind.

Völlige Ruhe im ganzen Gesichts-

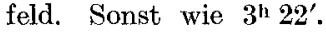

Dasselbe.

Dasselbe.

Bis auf geringe Blutbewegung in einer Vene völlige Ruhe.

Dasselbe.

$0,6 \% \mathrm{NaCl}$.

Völlige Ruhe.

Hier vermag die 1 proz. Hypophysisoptonlösung den Eintritt des Kreislaufstillstandes nicht zu verhindern oder zeitlich zubeeinflussen. Dagegen ist durch das Hypophysisopton das Kreislaufsbild in dem beschriebenen Sinne völlig geändert. Außerdem geht die Stase in der mit Hypophysisopton behandelten Schwimmhaut bedeutend schneller zurück, Befunde, dic also dem Hypophysisopton Gefäßerweiterung und Hemmung der Adrenalinwirkung als charakteristische Eigenschaften zuzuerkennen nötigen.

Ran. tempor. Protokoll IX.

\begin{tabular}{|c|c|}
\hline $\begin{array}{l}3^{\text {h }} 35^{\prime} \\
3^{\text {h }} 47^{\prime} \\
3^{\text {h }} 49^{\prime}\end{array}$ & $\begin{array}{l}4 \% \mathrm{Na}_{2} \mathrm{CO}_{3} . \\
0,6 \% \mathrm{NaCl} . \\
\text { Adrenalin } 1: 75000 .\end{array}$ \\
\hline $3^{\mathrm{h}} 50^{\prime}$ & $\begin{array}{l}\text { Die arteriellen Gefäße sind etwas } \\
\text { verengt. Der Kreislauf ist etwas } \\
\text { verlangsamt. }\end{array}$ \\
\hline
\end{tabular}

$3^{\text {h }} 51^{\prime} \quad$ Weitere Verlangsamung des Kreislaufes. Die Blutbewegung ist deutlich ruckweise.

$3^{\text {h }} 53^{\prime} \quad$ Die große Arterie ist nicht mehr sichtbar. Auch die übrigen Gefäße verengt. $Z$. T. besteht Stase, z. T. langsame Zirkulation, letztere besonders in den großen Venen und ihren Zuflüssen.
$4 \% \mathrm{Na}_{2} \mathrm{CO}_{3}$.
$0,6 \% \mathrm{NaCl}$.

Adrenalin $1: 75000+$ Corp. lut.opt. $2 \%$.

Keine Veränderung des Kreislaufes sowohl hinsichtlich der Schnelligkeit der Blutbewegung wie der Weite der Gefäße.

Dasselbe.

Dasselbe. 
Ran. tempor. Protokoll IX (Fortsetzung).

$3^{\text {h }} 58^{\prime} \quad$ Im größten Teil des Gesichtsfeldes

Dasselbe. vollständige Ruhe. Nur in wenigen Gefäßen sehr langsame Zirkulation.

$4^{\mathrm{h}} 3^{\prime} \quad$ Wesentliche Besserung des Kreislaufes. Nur in einem kleinen

Dasselbe.

Teil des Gesichtsfeldes besteht noch Stase, im übrigen nur wenig verlangsamte Blutbewegung.

$4^{\text {h }} 10^{\prime} \quad$ Kreislauf wieder völlig hergestellt. Dasselbe. Ran. tem por. Protokoll X.
$12^{\mathrm{h}} 8^{\prime}$
$12^{\mathrm{h}} 18^{\prime}$
$12^{\mathrm{h}} 20^{\prime}$
$12^{\mathrm{h}} 22^{\prime}$
$121127^{\prime}$
$12 \mathrm{~g}: 392^{\prime}$
Adrenalin $1: 75000+$ Corpus lut. opton $1 \%$ \%
Beiderseits Arterie verengt. Der ('esamtkreislauf ist etwas ver- langsamt.
Wis besteht noch ereringe Verenge- rung der Arterie. Dic Verlang- samung des Kreislanfes ist nur sehr gering.
Der Kreislaut ist wieder fast völlig normal. Kéne Gefäb- verengerung, nur unbedeutende. Verlangsamum der Zirkulation.

$12^{h} 38^{\prime}$

$12^{\mathrm{h}} 45^{\prime}$

Kreislauf normal, wie ror Beginm des Versuchs.

12 ly $49^{\prime}$ Dasselbe.

$12^{n} 57^{\prime}$ Dasselhe.
$4 \% \mathrm{Na}_{2} \mathrm{CO}_{3}$.

$0,6 \% \mathrm{NaCl}$.

Kreislauf erheblich rerlangsamt. Starke Verengerumg der Arterie.

In eine'n groBen Teil der Capillaren Stase. In der Arterie stark rerlangsamte Blutbewegung. Auch in der großen Vene Verlangsamung der Zirkulation, aber in geringerem Maße.

Wir $12^{\mathrm{h}} 32^{\prime}$.

Nur in der großen Vene Zirkulation; sonst völlige Ruhe.

$12^{\mathrm{h}} 49^{\prime}$ Wiederbeginn der arteriellen Zirkulation. Dieselbe ist aber nooh sehr stark rerlangsamt und erfolgt ruckweisc. $12^{\text {h }} 57^{\prime}$ Zeitweilig völlige Ruhe im ganzen Gesichtsfeld. Dann wieder ruckweise Zirkulation.

In beiden Verwuchen ist eine Hem mungder Adrenalinwirkung durch 1 bzw. 2proz. Corpus luteum-Opton erkennbar. Wir mörhten hierbei betonen. daßs der starken Wirksamkeit des Corpus luteum-Optons in den von uns früber beschicichenen Versuchen [besonders am Auge und überlebendem Frosehberz ${ }^{1}$ yowic am Oeso-

1) Abderhaliten und (iellhorn. Pflügers Arch. f. d. ges. Physiol. 182. 28. 1920. 
phagus $^{1}$ )] die Gefäßwirkung an der Schwimmhaut nicht entspricht. Vielmehr ließ sich in einer Anzahl von Versuchen, besonders mit etwas stärkeren Adrenalinlösungen, kcine sichere Wirkung nachweisen, ein Befund, der um so merkwürdiger ist, als das in den bisherigen Versuchen nur in geringem Maße wirksame Ovarialopton konstant und in viel stärkerem Maße der Adrenalinwirkung am Kreislauf in der Schwimmhaut entgegenwirkte.

Es bleiben nunmehr noch die Versuche, die mit Testis- und Placentaopton angestellt sind, zu besprechen. Von beiden verwendeten wir fermentativ abgebaute (N-Präparate) sowie durch Hydrolyse mit 10 proz. Schwefelsäure dargestellte Präparate (S-Präparate). Wir haben eine außerordentlich gxoße Anzahl von Versuchen angestellt, um die Frage zu entscheiden, ob durch die verschjedene Darstellung Körper von verschiedenartiger Wirkung gebildet werden. Hatten doch neben Gemeinsamkeiten z. B. dic Optone der Placenta (S und N) auch verschiedene Wirkungen am Herzstreifen ergeben and ferner wurde in zahlreichen Versuchen festgestellt, daß nur dem durch Hydrolyse mit 10 proz. Schwefelsäure dargestellten Testisopton die auf die Automatie des nicht schlagenden Herzstreifens anregende Wirkung zukommt. Die Versuche an der Schwimmhaut führten zu dem Resultat, daß Testis $\mathrm{N}$ ebenso wie Placenta s keine sichere Beeinflussung des Kreislaufes zeigt. Vichmehr glauben wir den ungleichmäßigen Ausfall einer großen Reihe von Versuchen sowie dem Mißlingen eines Doppelversuches an ein und demselben Präparat, der als Experimentum crucis angesehen werden könnte, im Sinne der Unwirksamkeit der genannten Präparate deuten zu müssen. Dagegen konnten mit Placenta $\mathbf{N}$ und Testis $\$$, wie die Protokolle XI und XII zeigen, eindeutige Versuche ausgeführt werden, die die Hemmung der Adrenalinwirkung durch die genannten optone dartun.

Ran. tom p. Protokoll XI.

\begin{tabular}{|c|c|c|}
\hline $11^{\mathrm{h}} 15^{\prime}$ & Natr. carbon $4 \%$ & Natr. carbon. $4^{\circ} \%$ \\
\hline $11^{\mathrm{h}} 25^{\prime}$ & $0,6 \% \mathrm{NaCl}$ & $0,6^{\circ} \% \mathrm{NaCl}$ \\
\hline $11^{\mathrm{h}} 26^{\prime}$ & $\begin{array}{l}\text { Adrenalin } 1: 40000+2 \frac{1}{2} \% \text { Pla- } \\
\text { centa N-Opton. }\end{array}$ & Adrenalin $1: 40000$. \\
\hline $11^{\text {h }} 27^{\prime}$ & $\begin{array}{l}\text { Gefäße erweitert. Kreislauf ctwas } \\
\text { beschleunigt. }\end{array}$ & $\begin{array}{l}\text { Arterie stark verengt, Kreis ver- } \\
\text { langsamt. }\end{array}$ \\
\hline $11^{\mathrm{h}} 28^{\prime}$ & $\begin{array}{l}\text { Sehr stark beschleunigter Kreislauf } \\
\text { mit erweiterten Gefäßen. }\end{array}$ & $\begin{array}{l}\text { In der großen Arterie ganz lang- } \\
\text { same, ruckweise Blutbewegung. } \\
\text { In den Capillaren und kleinen } \\
\text { Arteriolen vollständige Ruhe. } \\
\text { Die große Vene zeigt noch gute } \\
\text { Zirkulation. }\end{array}$ \\
\hline
\end{tabular}

1) Abderhalden und Gellhorn. Pfliger's Arch. f. d. ges. Physiol. 18g, 243. 1921 . 


\section{Ran. tempor. Protokoll XI (Fortsetzung).}

$11^{\mathrm{h}} 27^{\prime}$ In der großen Vene nur sehr langsame, ruckweise Blutbewegung. Die große Arterie ist fast nicht sichtbar. Sonst völlige Ruhe.

$11^{\mathrm{h}} 30^{\prime} \quad$ Dasselbe. Auch Capillaren erweitert, strotzerd mit Blut gefüllt.

$11^{\mathrm{h}} 32^{\prime}-11^{\mathrm{h}} 40^{\prime}$ Dasselbe.

$11^{\text {h }} 43^{\prime}$ u. Kreislauf normal, nicht mehr be$11^{\text {h }} 50^{\prime}$ schleunigt. Gefäße von normaler Weite.

$11^{\mathrm{h}} 57^{\prime}$ Dasselbe.

$12^{\mathrm{h}} 5^{\prime} \quad$ Dasselbe.

$12^{\mathrm{h}} 6^{\prime} \quad 0,6^{\circ}{ }_{0}^{\prime} \mathrm{NaCl}$.

loh $y^{\prime}$ Kreislauf normal.

12"12' Dassetbe.

$12^{\mathrm{h}} 15^{\prime}$ Beiderseits normaler Kreislauf.

$12117^{\prime} \quad 4_{0}^{\prime \prime} \mathrm{Na}_{2} \mathrm{CO}_{3}$.

12. $23^{\prime} \quad 0,6^{\prime \prime} \mathrm{O}$ NaCl.

1.21 $24^{\prime}$ Artrenalin $1: 40$ (m)

1.21355 In der groiken Arterie Nitilstand der Blutbowegung. Fionst gutr. Kreislauf.

1:2067" Starke Verlangsamung anch in den Venen und Capillaren.

I $2427^{\prime} \quad$ Im ganzen Gesichtsfeld fast völlige Fuhe. Nur in einigen kleineren Tenen langsame Zirkulation.

l2" $29^{\prime} \quad$ Vollige liuhe im ganzen Gesichtsfold.

12]" 34' Wierlerheginn der Zirkulation. In einem Teil der Capillaren urst kicineren Arterien und Vemen noch livhe. In abrigen sehr langsame, ruckweise Bluthowegung.

$12^{\mathrm{h}} 45^{\prime}$ Wieder vällige Ruhe im ganzen resichtsfold.

1h $5^{\prime}$ Dasselhe.

Wh 15' last normaler Kreislauf. Nur dic großbe Arterie ist deutlich verengt. und zeigt langsanue Strömung.

Minimale Bewegung des Blutes in der großen Vene. Arterio nicht mehr sichtbar. Sonst völlige Ruhe.

Völlige Ruhe im ganz. GesichtsfeJd.

Dasselbe.

Die große Vene und ein Teil ihrer kleineren Äste zeigt langsame Zirkulation. Sonst Ruhe.

In den Venen normale Zirkulation. Sonst Ruhe.

(0. $6^{\circ}$;) Nar!1.

In der groben Arterie und Vene und einem Teil der (apillaren normale Zirkulation. Arterie noch derutlied verenst.

Writere Besserung des Gresant. kreislanfes.

$4^{\circ}, \mathrm{Na}_{2}(\mathrm{O})$

(0, (6), $\mathrm{Nar}$ (I)

Adrenalin 1: 400000 - I Iacentia N-1)ptum 2 $1 \%$ "..

Kreislauf nomal.

Dasiselbe.

Dasselloe.

Korislauf unter Erweiterung der Gefäbe deutlich beschleunist. Dasselbe.

Kreislauf pormal, nicht meht heschleunigt.

Dassello.

Dasselles. 
Die Kreislaufswirkung von Placenta N-Opton sei auch durch ein Mikrophotogramm illustriert, das aus dem Versuche des Protokolls XI stammt. Während unter der Einwirkung des Adrenalins sich die Gefäße sehr stark verengern und blutleer werden (Abb. 21), so daß sie oft nur schwer wahrzunehmen sind, bewirkt der Zusatz von Placenta N.Opton nicht nur eine Hemmung der vasoconstrictorischen Adrenalin-

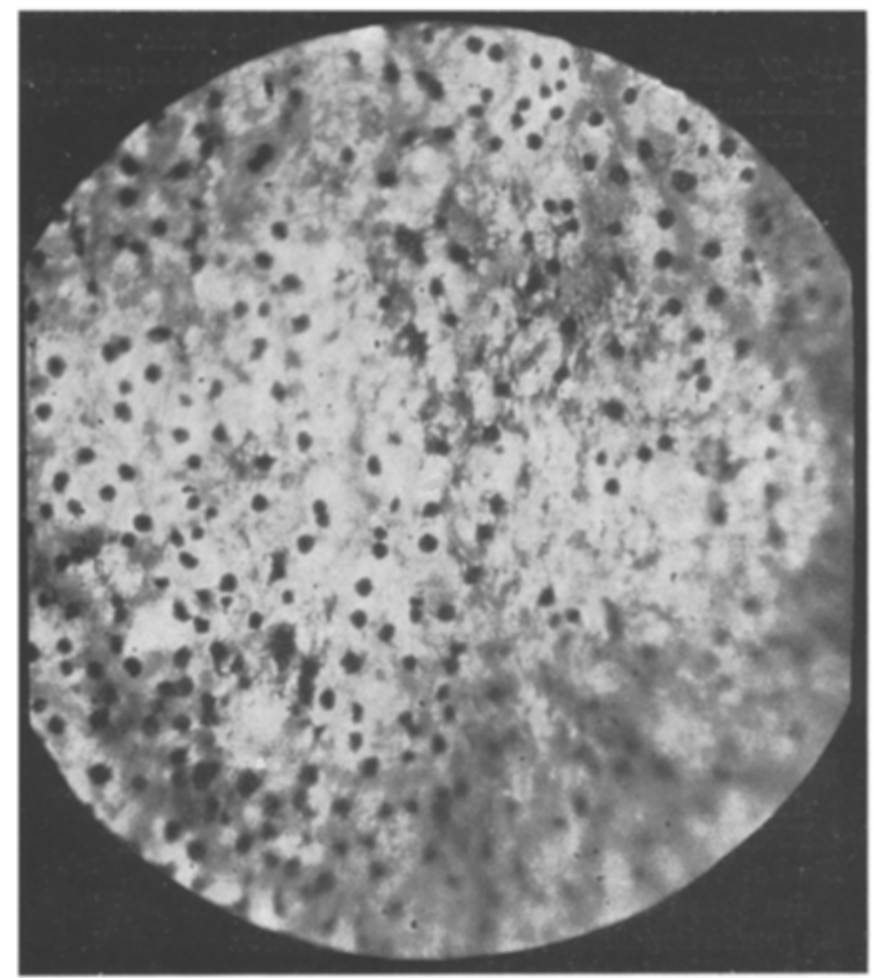

Abb. 21.

Rana temporaria. Mikrophotogramm der linken Schwimmhaut nach Einwirkung von Adrenalin $1: 40000$.

wirkung, sondern ruft direkte Erweiterung der Gefäße, insbesondere der Capillaren und kleineren Arteriolen hervor, die ganz prall mit roten Blutkörperchen gefüllt sind (Abb. 22). Dabei möchten wir betonen, daß diese Wirkung nicht etwa speziell für Placenta $N$ charakteristisch ist, sondern in mehr oder minderem Maße auch den übrigen Optonen zukommt, die die Adrenalinwirkung zu hemmen oder zu vermindern imstande sind. 
Ran. temp. Protokoll XII.

\begin{tabular}{|c|c|c|}
\hline $2^{\mathrm{h}} 10^{\prime}$ & $4 \% \mathrm{Na}_{2} \mathrm{CO}_{3}$ & $4 \% \mathrm{Na}_{2} \mathrm{CO}_{3}$ \\
\hline $20^{\prime}$ & $0,6 \% \mathrm{NaCl}$. & $0,6^{\circ} \% \mathrm{NaCl}$ \\
\hline $2^{\text {h }} 22$ & Adrenalin 1: 40000. & $\begin{array}{l}\text { Adrenalin } 1: 40000+\text { Testis } \\
\text { Opton } 1 \% \%\end{array}$ \\
\hline $\begin{array}{l}\text { h } 24^{\prime} \\
\text { hn } 25^{\prime}\end{array}$ & $\begin{array}{l}\text { Beiderseits Kreislauf verlangsamt. } \\
\text { In dem größten Teil des Gesichts- } \\
\text { feldes Stase. }\end{array}$ & $\begin{array}{l}\text { Nirgends Stase. Kreislauf } \\
\text { etwas verlangsamt. }\end{array}$ \\
\hline
\end{tabular}

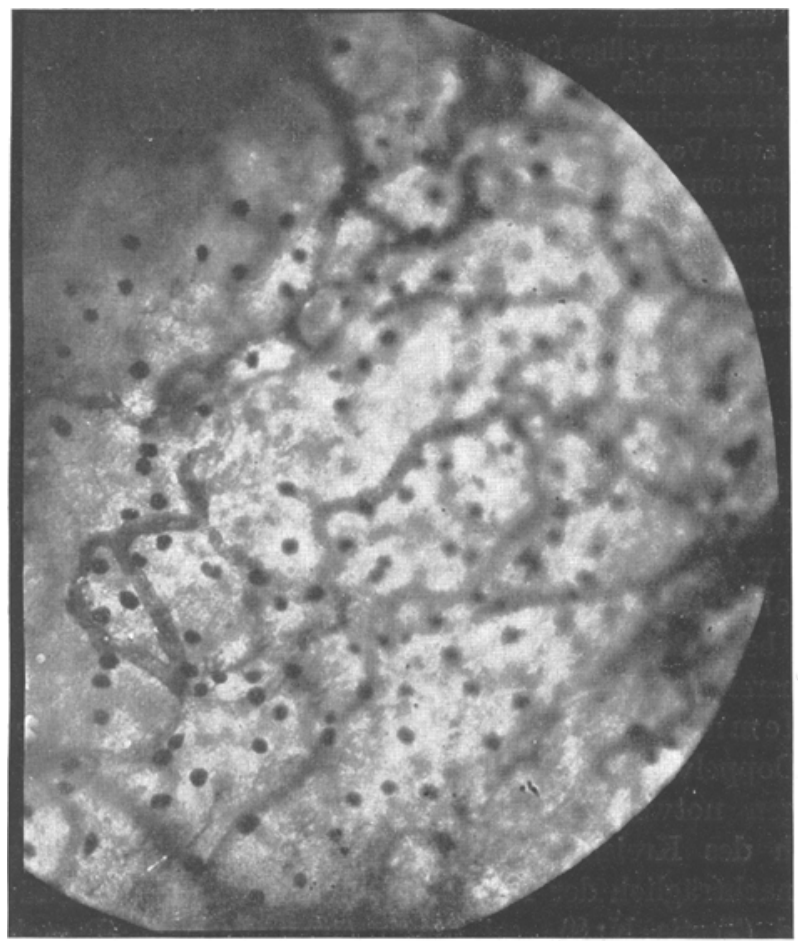

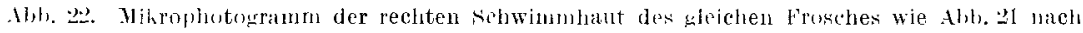

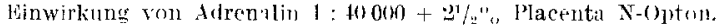

2h 27' Nur in dereroben Fene sehr langsame Blutbewegung. SonstRuhe im ganzen Gesichtisfeld.

$2{ }^{2}{ }_{30} \quad$ Dasselbe.

2h $: 92^{\prime} \quad$ Völlige Ruhe im ganz. Gesichtsfold.

?h $42^{\prime}$ Dasselhe.

$2^{\mathrm{h}} 44^{\prime} \quad 0,6^{\prime \prime} \% \mathrm{NaCl}$.

$2^{1 !} 46^{\prime}$ In der grolion Arterie ganz langsamc, rhythmische Blutbewegungen. Somst vallige Ruhe.
Nur geringe Voplangsamung des Kreislaufes.

Dasselbe.

Dasselbe.

Kreislauf nur wenig verlangsamt. In einem kleinen Teil des Gesichts. feldes Stase.

$0,6 \div$ NaC?.

Zirkulation erheblich beschleunicgt, wieder völlig normal. 
Ran. tempor. Protok oll XII (Fortsetzung).

$2^{\text {h }} 50^{\prime} \quad$ Kreislauf beiderseits wiederhergestellt.

$2^{\mathrm{h}} 55^{\prime} \quad 4_{\%}^{\circ} \mathrm{Na}_{2} \mathrm{CO}_{3}$

$3^{\text {h }} 5^{\prime} \quad 0,6 \%$ NaCl.

$4 \% \mathrm{Na}_{2} \mathrm{CO}_{3}$

$3^{\text {h }} 8^{\prime} \quad$ Adrenalin $1: 40000+$ Testis $s$ - Adrenalin $1: 40000$. opton $1^{\circ}$;

$3^{\text {h }} 9^{\prime} \quad$ In zwei Venen noch Zirkulation; sonst Stase. Keine Verengerung der Gefäße.

$3^{\text {h }} 11^{\prime}$ u. Beiderseits völlige Ruhe im ganzen

$3^{\text {h }} 13^{\prime}$ Gesichtsfeld.

$3^{\text {h }} 20^{\prime}$ Wiederbeginn der Zirkulation in Völlige Rube. zwei Venen. Sonst Ruhe.

$3^{\text {h }} 42^{\prime} \quad$ Fast normaler Kreislauf. Nirgends Stase. Nur unbedeutende Verlangsamung der Zirkulation.

$4^{\mathrm{h}} \quad$ Normaler Kreislauf.

$4^{\text {h }} 25^{\prime}$ Dasselbe.

Völlige Ruhe im ganzen Gesichtsfeld. Gefäße sehr eng.

Dasselbe.

Dasselbe.

Kreislauf wiederhergestellt. Nirgends Stase. Aber noch deutliche Verlangsamung der Zirkulation.

Aus dem Versuche mit Placenta N-Opton ist besonder's hervorzuheben, daß nicht allein Adrenalin 1:40000 unwirksam gemacht wurde, sondern außerdem eine bedeutende Kreislaufsbeschleunigung und Erweiterung der Gefäße eintritt. Dabei ist bemerkenswert, daß der Erfolg 1 proz. Placenta N-Optonlösungen bereits ein sehr unsicherer ist.

Der Hemmung der Adrenalinwirkung durch Testis $\mathrm{S}$, die aus dem Doppelversuch des Protokolls XII hervorgeht, scheint nichts hinzuzufügen notwendig. Die interessante Tatsache aber, daß das hinsichtlich des Kreislaufes inaktive Testis N-Opton wirksam wird. sobald es nachträglich der Hydrolyse mit 10 proz. Schwefelsäure unterzogen wird (Testis N. S), sei durch einen Versuch (Protokoll XIII) näher illustriert.

Ran. tempor. Protokoll XIII.

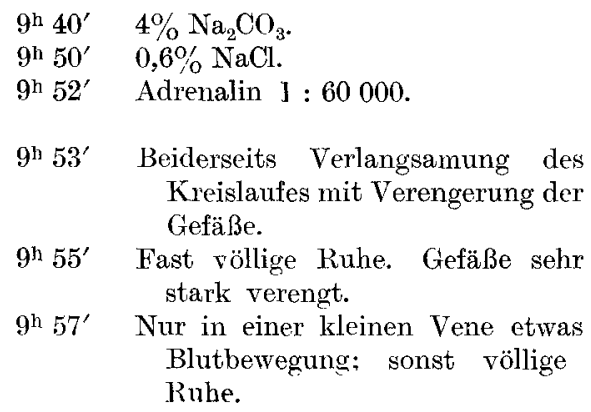

$9^{\text {h }} 55^{\prime} \quad$ Fast völlige Ruhe. Gefäße sehr stark verengt.

$9^{\mathrm{h}} 57^{\prime} \quad$ Nur in einer kleinen Vene etwas Blutbewegung; sonst völlige Rube.

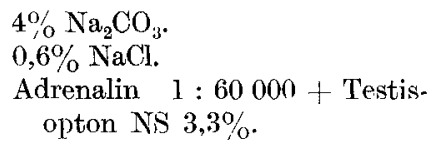

Kreislauf etwas verlangsamt, zum kleinen Teil Stase.

Gefäße nicht mehr verengt, gut: mit Blut gefüllt. Kreiclauf nur unbedeutend verlangsamt. 


\section{Ran. tempor. Protokoll XIII (Fortsetzung).}

$10^{\text {h }}$ Fast völlige Ruhe.

$10^{\mathrm{h}} 5^{\prime} \quad 0,6 \mathrm{NaCl}$.

$10^{\mathrm{h}} 7^{\prime}$ Wiederbeginn der Zirkulation in der großen Arterie und Vene. Im größten Teil der Capillaren noch Stase.

10: 12' Keine weitere Besserung.

$10^{\mathrm{h}} 22^{\prime} \quad$ Kreislauf völlig wiederhergestellt.

$10^{\mathrm{h}} 23^{\prime} \quad 4 \% \mathrm{Na}_{2} \mathrm{CO}_{3}$.

10 $33^{\prime} \quad 0,6 \% \mathrm{NaCl}$.

$10 \mathrm{~h} 35^{\circ}$ Adrenalin $1: 60000+$ Testis NS $3,3 \%$.

Inin $36^{\circ}$ Beiderseits Verlangsamung des Kreislaufes.

L(1:138' Verlangsamung fast völlig beseitigt. Gefäße weit, strotzend mit Blut gefüllt. Nirgends Stase.

$10{ }^{1} 42^{\prime}$ 'Zirkulation verlangsamt, ruck weise. Sämtliche Gefäße weit und gut gefiullt. In einem Teil des Gesichtsfoldes Stase.

$14+1+5^{\prime} \quad\left(0,6^{\circ} \% \mathrm{NaCl}\right.$

10": $49^{\prime}$ Kreislauf wiederhergestellt. Nirgends Stase. Im ganzen noch geringe Verlangsamung der Blutbeweging.

ththe $\tilde{n}^{\prime} \tilde{n}^{\prime}$ Kreislauf beiderseits fast normal. Gefäße weit. Nur geringe Stromverlangsamnng.
Nirgends Stase.

Kreislauf fast normal.

$0,6 \% \mathrm{NaCl}$.

Kreislauf normal.

Kreislauf normal.

Kreislauf normal.

$4 \% \mathrm{Na}_{2} \mathrm{CO}_{3}$.

$0,6 \% \mathrm{NaCl}$.

Adrenalin $1: 60000$.

Fast völlige Ruhe, Nirr in der großen Vene sehr langsame Virkulation. Capillaren, Arteriolen und kleine Arterien ganz eng und blutlecr.

Völlige Ruhe bis auf die ruck. weise, sehr langsame Zirku. lation in der großen Vene. Gesichtsfeld ganz blal.3 und blutleer.

$0,6 \% \mathrm{NaCl}$.

In der großen Vene notmale Zirkulation. Sonst Puhe.

Endlich sei noch erwähnt, daß auch das Thymusopton dic Invenalinwirkung hemmt.

Ran. tempor. Protokoll Xrr.

\footnotetext{
$2^{i} b^{\prime} \quad$ Natr. carbon $4 \%$.

$2^{\text {h }} 16^{\prime} \quad 0,6 \% \mathrm{NaCl}$.

2 : $17^{\prime}$ Adrenalin 1:30000+20; Thymusopton.

$2^{\text {hn }} 18^{\prime} \quad$ Kreislauf unverändert.
}

Natr. carbon. $4 \%$

$0,6^{\prime} \mathrm{NaCl}$.

Adrenalin 1:30000.

Arterie stark verengt. Zirkulation im ganzen Gesichtsfeld deutlich verlangsant.

$12^{\mathrm{h}} 18^{\prime} 30^{\prime \prime}$ Arterie fast nicht mehr sichtbar. In dor großen Vene riickläufige Bewegung des Blutes. 
82 E. Abderhalden u. E. Gellhorn: Weitere Untersuchungen über die

$$
\text { Ran. tempor. Protokoll XIV (Fortsetzung). }
$$

$12^{\mathrm{h}} 19^{\prime} \quad$ Im ganzen ist der Kreislauf verlangsamt, in einem Teil der Capillaren Stase, in der großen Arterie Zirkulation kaum verlangsamt.

$12^{\mathrm{h}} 22^{\prime}$ Auch in der Arterie etwas verlangsamte Blutbewegung. Capillaren wie $12^{\mathrm{h}} 19^{\prime}$. In größeren und kleineren Venen langsame Zirkulation in normaler Richtung.

$12^{\mathrm{h}} 25^{\prime}$ Fast derselbe Befund. Nur in einigen Capillaren wieder beginnende Strömung.

$12^{\mathrm{h}} 26^{\prime} \quad$ Kreislauf erheblich gebessert. Nirgends Stase. Zirkulation nur wenig verlangsamt.

$12^{\mathrm{h}} 28^{\prime} \quad$ Kreislauf fast völlig normal. Gefäße noch etwas verengt.

$12^{\text {h }} 29^{\prime} \quad 0,6 \% \mathrm{NaCl}$.

$12^{\mathrm{h}} 29^{\prime} 80^{\prime \prime}$ Kreislauf bedeutend beschleunigt, wieder völlig normal. Gefäße wieder weiter. $12^{\mathrm{h}} 19^{\prime}$ Völlige Ruhe im ganzen Gesichtsfeld.

12h $22^{\prime}$ Dasselbe. Arterie völlig verschwunden.

Völlige Ruhe.

Völlige Ruhe.

Völlige Ruhe.

$0,6 \% \mathrm{NaCl}$.

Ruhe unverändert.

Ran. tempor. Protokoll XV.

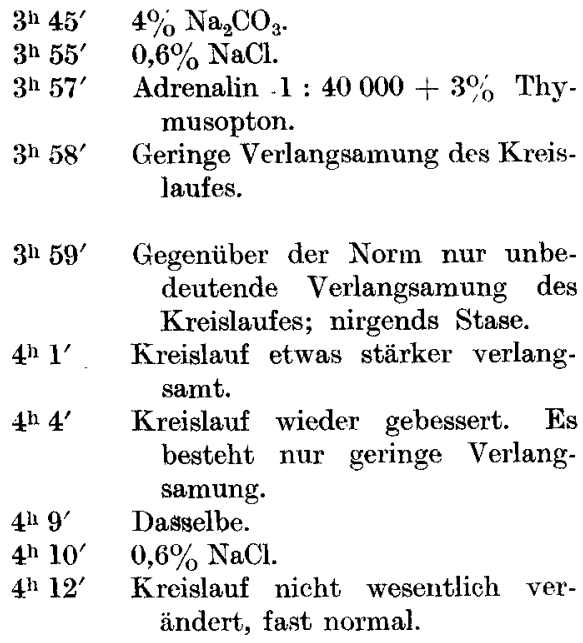

$4^{\text {h }} 12^{\prime}$ Kreislauf nicht wesentlich verändert, fast normal.

$4 \% \mathrm{Na}_{2} \mathrm{CO}_{3}$. $0,6 \% \mathrm{NaCl}$.

Adrenalin 1:40000.

Völlige Ruhe im ganzen Gesichtsfeld mit starker Gefäßverengerung.

Dasselbe.

Dasselbe.

Dasselbe.

Dasselbe.

$0,6 \% \mathrm{NaCl}$.

In den großen Arterieu und Venen Wiederbeginn der Zirkulation. Sonst noch völlige Ruhe.

Ineinigen Versuchen gelang es auchdirektdurch Thymusopton eine geringe GefäBerweiterung zu erzielen. 
Zusammenfassung.

1. Mit durch Hydrolyse mit 10 proz. Schwefelsäure gewonnenem Testisopton (S) gelingt es, die Automatie nicht. schlagender Herzstreifen (auch Herzspitze) zu erregen. Hoden, die durch Fermente abgebaut sind (N), haben keine Wirkung. Nachträgliche Hydrolyse dieser Präparate mit 10 proz. Schwefelsäure führen wieder zu der charakteristischen Wirkung.

2. Die Wirkung des Testishydrolysats wird durch Entfernung deralkohollöslichen Produkteverstärkt (Extrakt ()).

3. Placenta N-Opton bewirkt am Straubschen Präparat nach vorübergehender Pulsverkleincrung ejne Vergrößerung der Kontraktionen. Placentas.Opton besitzt lediglich eine negative inotrope Wirkung. In Übereinstimmung mit früheren Frgebnissen kommt auch diesen Optonen am Herzstreifen eine negativ chronotrope Wirkung zu.

4. Schilddräsen- und Strumahydrolysat erregen die Automatic und den Tonus des überlebenden Froschoesophagus. In gleichen Konzentrationen wirkt Placenta N. und S-Opton stark lähmend. In schwächeren Lösungen hat Placenta S-Opton Vergröberung der Kontraktionen zur Folge.

5. Der Lxtrakt ( a as Hodenhydrolysat regt auch die Automatie des nicht schlagenden Oesophagus an.

6. Dir Optono aus Schilddrüse, Hypophyse, Thymus, Grar, Placenta N, Testis s besitzen eine mehr oder minder starke capillar-und z. T. a uch artericnerweiternde Wirkung. Dic Adrenalinwirkung wird durch die genannten optone gehemmt oder vermindert.

Es geht aus den gemachten Feststellungen mit gröBter Wahrscheinlichkeit hervor, da 3 die Inkrete als Regulatoren des Capillarmechanismus eine bedeutsame Rolle spielen. Es wird darauf hingewiesen, daß in Zukunft diesem Zusammenwirken von Organ und Capillarsystem a uch bei der Feststcllung des Wesens von Ausfallserscheinungen beim Versagen von inkretstoffe liefernden Geweben vermehrte A ufmerksam keit zuzuwenden ist. 